Portland State University

PDXScholar

11-14-1995

\title{
Complexity Properties of the Cellular Automaton Game of Life
}

Andreas Rechtsteiner

Portland State University

Follow this and additional works at: https://pdxscholar.library.pdx.edu/open_access_etds

Part of the Physics Commons

Let us know how access to this document benefits you.

\section{Recommended Citation}

Rechtsteiner, Andreas, "Complexity Properties of the Cellular Automaton Game of Life" (1995).

Dissertations and Theses. Paper 4928.

https://doi.org/10.15760/etd.6804

This Thesis is brought to you for free and open access. It has been accepted for inclusion in Dissertations and Theses by an authorized administrator of PDXScholar. Please contact us if we can make this document more accessible: pdxscholar@pdx.edu. 


\section{THESIS APPROVAL}

The abstract and thesis of Andreas Rechtsteiner for the Master of Science in Physics were presented November 14th, 1995, and accepted by the thesis committee and the department.

COMMITTEE APPROVALS:

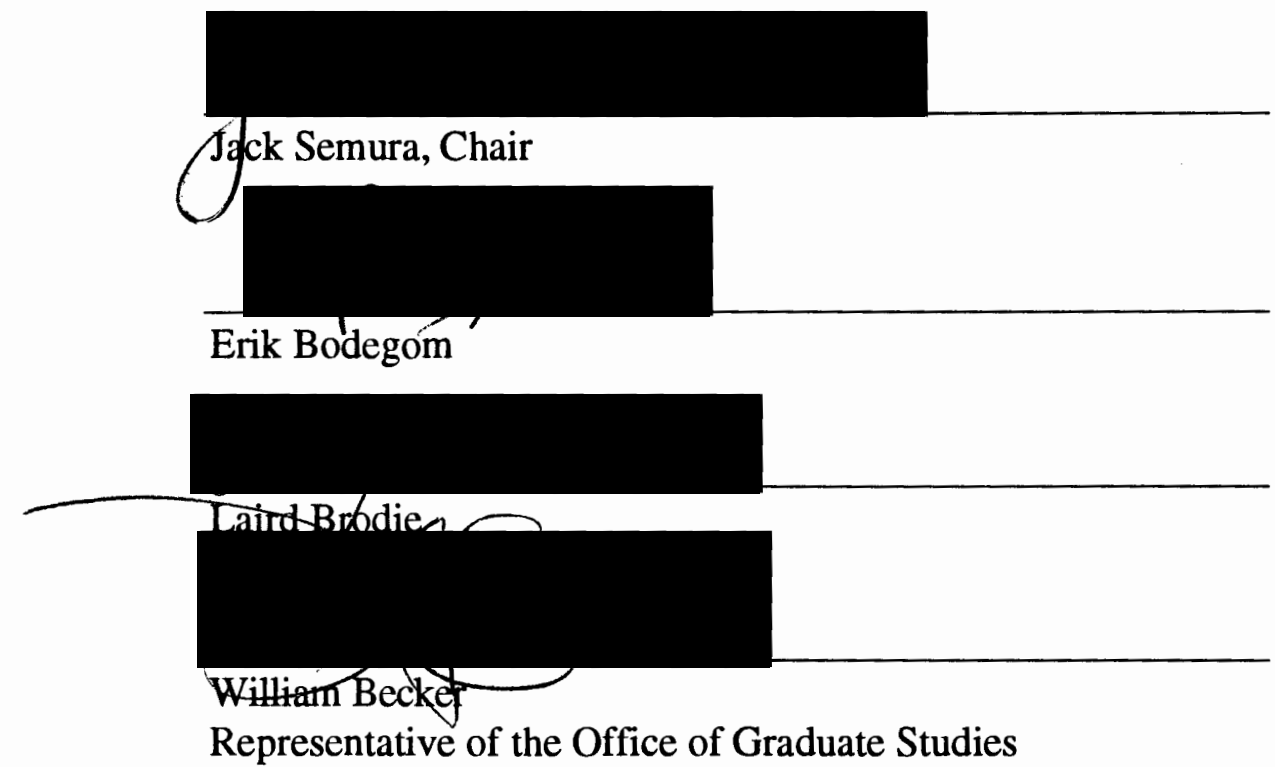

DEPARTMENT APPROVAL:

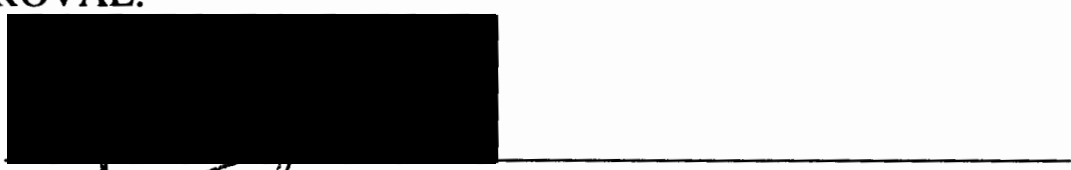

Erik Botegom, Chair

Department of Physics

$* * * * * * * * * * * * * * * * * * * * * * * * * * * * * * * * * * * * * * * * * * * * * * * * * * * * * * * * * * * * * * * *$

ACCEPTED FOR PORTLAND STATE UNIVERSITY BY THE LIBRARY

by on Selkerntur 1995 


\begin{abstract}
An abstract of the thesis of Andreas Rechtsteiner for the Master of Science in Physics presented November 14, 1995.
\end{abstract}

Title: Complexity Properties of the Cellular Automaton Game of Life

The Game of Life is probably the most famous cellular automaton. Life shows all the characteristics of Wolfram's complex Class IV cellular automata: long-lived transients, static and propagating local structures, and the ability to support universal computation.

We examine in this thesis questions about the geometry and criticality of Life. We find that Life has two different regimes with different dimensionalities. In the small scale regime Life shows a fractal dimensionality with $D_{s}=0.658$ and in the large scale regime $D_{l}=2.0$, suggesting that the objects of Life are randomly distributed. We find that Life differentiates between different spatial directions in the universe. This is surprising because Life's transition rules do not show such a differentiation. We find further that the correlations between alive cells extend farthest in the active period and that they decrease in the glider period, suggesting that Life is sub-critical. Finally, we find a size-distribution of active clusters which does not depend on the lattice size and amount of activity, except for the largest clusters. We suggest that this result also indicates that Life is sub-critical. 


\title{
Complexity Properties of the Cellular Automaton Game of Life
}

\author{
By \\ Andreas Rechtsteiner
}

A thesis submitted in partial fulfillment of the requirements for the degree of

MASTER OF SCIENCE

in

PHYSICS

Portland State University

1995 


\section{Acknowledgments}

First and most of all I would like to thank my advisor, Prof. Jack Semura. It was a great pleasure, personal and professional, to work with him. I have never met before a physics professor so excited about his subject. His constant wondering about physics, and everything in the world, and his never ending production of physical ideas was inspiring and helped me to overcome some frustrations with my work. Without his knowledgeable advise this thesis would not have been possible. I am also very grateful for his help in finding me a teaching assistantship, without which I would not have been able to finish my work.

I would also like to thank Prof. William Becker and all the people in the Center for Science Education for offering me the chance to work with them as a teaching assistant. Without the financial support of the teaching assistantship I would not have been able to finish this thesis. I am thankful for the many positive experiences I have had during the last year and for the never-ending patience of some people in CSE who are still trying to teach me the subtleties of American humor.

I want to thank all the people in the physics department as well. Whenever I came with a question or a problem I found support and help.

I want to thank my friend Martin Heni who had the misfortune of visiting me from Germany while I was finishing my thesis. His suggestions and the discussions with him were of great help to me. I also want to thank Eddie Smith who has gratefully offered to help me overcome my obstacles with the English language. 
Finally, I want to thank my family and all my friends for their emotional support. I want to thank especially Rossana Viteri-Burbano whose friendship has helped me so much in difficult situations over the last two years. 


\section{Table of Contents}

Table of Contents $\quad 1$

Chapter 1 Introduction 3

Chapter 2 Cellular Automata 6

2.1 Definition of Cellular Automata $\quad 6$

2.2 Computation in Cellular Automata $\quad 8$

2.3 Von Neumann's Work on Self-Replication 9

Chapter 3 Complexity and Criticality in Cellular Automata 12

3.1 Wolfram's Classification of Cellular Automata 12

3.2 Computation in Class IV Universes $\quad 14$

$\begin{array}{ll}\text { 3.3 Langton's } \lambda \text { parameter } & 16\end{array}$

3.4 Continuos Phase Transitions 20

3.5 Correlation Functions 23

$\begin{array}{ll}\text { 3.6 Self-Organized Criticality } & 25\end{array}$

Chapter 4 The Cellular Automaton Game of Life 29

$\begin{array}{llr}4.1 & \text { Introduction } & 29\end{array}$

4.2 Dynamical Properties of Life 32

4.3 Universal Computation in Life 34

4.4 Self-Organized Criticality in Life $\quad 37$ 
Chapter 5 Results and Discussion $\quad 42$

5.1 General Methodology used in Simulations 42

5.2 Distribution of Objects in the Final Steady State of Life 43

5.3 Fractal Box Count Dimensionality of the Final Steady State of Life 43

5.3.1 Box Count Method 44

5.3.2 Theoretical Models 46
A. Large Scale Regime 46
B. Small Scale Regime 47
C. Dividing Scale for Large and Small Scale Regime 53

5.3.3 Experimental Box Count Results $\quad 54$

5.4 Correlations between alive Cells $\quad 59$

5.5 Geometrical Properties of Life 62

5.6 Size-Distribution of Active Clusters 66

$\begin{array}{lll}5.7 \text { Summary of Results } & 71\end{array}$

$\begin{array}{ll}\text { Bibliography } & 74\end{array}$ 


\section{Chapter 1}

\section{Introduction}

One of the great unsolved questions in science is how the complexity in our world, with its self-organizing and living systems, could evolve. The second law of thermodynamics describes the world as evolving from order to disorder, but biological and social evolution shows the complex evolving from the simple. Some progress has been made regarding this question. We know now that nonequilibrium, the flow of matter and energy, can be a source of order. In the last decades scientific interest in the many remaining questions has increased. The simulation possibilities arising from growing computing power have led to a new interdisciplinary research field in science called complexity ${ }^{a}$

The goal in complexity research is to find basic, abstract, and general concepts which can be applied to systems in many different fields like chemistry, biology, sociology or economics. As early as 1972, Philip. W. Anderson, a condensed matter physicist, Nobel laureate, and one of the founders of the Santa Fe Institute, published a famous article which contains some of the main ideas of complexity [1]. He developed a theory of a hierarchical structure in science where the laws and concepts found at a lower level are applied to questions in science on the next higher level. Elementary

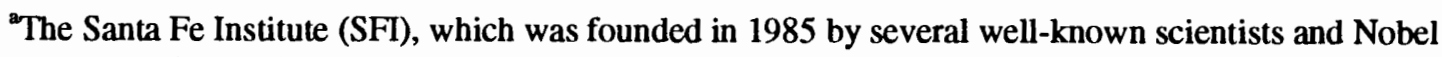
laureates from different scientific fields, is probably the best known place where complexity research is performed (see Mitchell Complexity, 1992).
} 
particle physics, many-body physics, chemistry, and molecular biology are examples of different consecutive levels. Anderson argues further that the laws from a lower level are never sufficient to explain all phenomena on the next higher level and that therefore at every level entirely new laws, concepts and generalizations are necessary. But this view is not generally accepted [2]. Complexity takes Anderson's view and tries to explain how emergence and self-organization happens and what makes new concepts and laws necessary on each level.

In this work, we examine the properties of a complex cellular automaton known as the Game of Life. The Game of Life is a cellular automaton that was invented by the mathematician John Horton Conway in 1970. Life was popularized by several monthly columns of Martin Gardener in Scientific American [3] and became famous because of its complicated local structures and complex global dynamics that arise out of very simple rules. Although Life was at the beginning basically a toy for computer programmers and game lovers, it soon became apparent that there was much more to this cellular automaton than just being a computing time killer. During the following years Life has been the subject of intense scientific research, some of it controversial. Because of its properties, Life is an often-used model for complexity. However, there are questions about complexity as well as Life that have not been resolved yet. In this thesis we will try to organize the major findings about Life, and examine some of these questions through our simulations in a new perspective. We also want to address the most controversial question about Life, if it is a self-organizing critical system. In 
addition we will present some new properties of Life: the fractal box count dimensionality of the final steady state and results about spatial correlations in Life.

In Chapter 2, we introduce cellular automata with a formal definition and give a short summary of von Neumann's work on self-replicating systems and cellular automata. In Chapter 3, we present the major results of the work on complexity and criticality in cellular automata. We will also briefly discuss critical phenomena and the related concept of self-organized criticality. In Chapter 4, we introduce the Game of Life and present the most important findings about this cellular automaton. Chapter 5 contains the results and discussions of our simulations. 


\section{Chapter 2}

\section{Cellular Automata}

\subsection{Definition of Cellular Automata}

Cellular automata can be described as artificial programmable "universes", which are discreet in time and space. The physics of these logical universes is a deterministic, local physics. Local means that the state of a cell at time $t+1$ is only a function of its own state and the states of the cells in a certain neighborhood at time $t$. Deterministic means that once a local physics and an initial state of a cellular automaton has been chosen, its future evolution is uniquely determined.

Formally, a cellular automaton is a $D$-dimensional lattice with a finite automaton residing at each lattice site. At any given time and in any given cell, the automaton can be in only one of a finite number of states. Conway's Game of Life, for example, has just two states, also named living and dead, and is therefore called a twostate cellular automaton. Each cell of the automaton takes as input the states of the cells within some finite local region of the cell, defined by a neighborhood template $N$, where the dimension of $N$ has to be smaller than or equal to $D$. The size of the neighborhood template, $|N|$, is just the number of lattice points covered by $N$. By convention, an automaton is considered to be a member of its own neighborhood. 
Figure 1 shows the two most common neighborhoods for two-dimensional cellular automata.

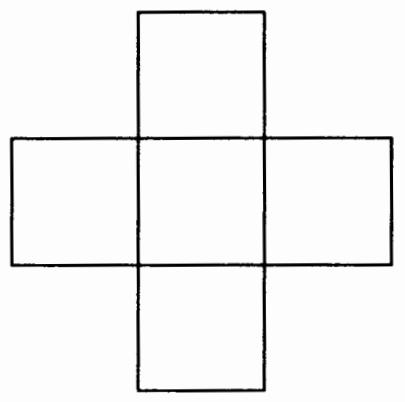

five cell, or von Neumann neighborhood

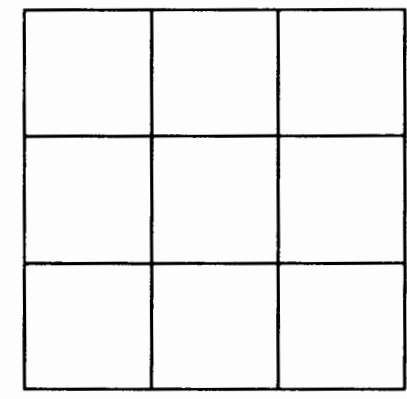

nine cell, or Moore neighborhood

Fig. 1: Two characteristic neighborhoods for two dimensional cellular automata

Each automaton consists of a finite set of cell states $\mathrm{E}$, a finite input alphabet $\alpha$, and a transition function $\Delta$, which is a mapping from the set of neighborhood states to the set of cell states. Letting $\mathrm{N}=|N|$ :

$$
\Delta: \mathrm{E}^{\mathrm{N}} \rightarrow \mathrm{E}
$$

The state of a neighborhood is the cross product of the states of the automata covered by the neighborhood template. Thus, the input alphabet $\alpha$ for each automaton consists of the set of possible neighborhood states: $\alpha=E^{N}$. Letting $K=|E|$, the number of cell states, the size of $\alpha$ is equal to the number of possible neighborhood states:

$$
|\alpha|=|\Delta|=\left|E^{N}\right|=K^{N}
$$


To define a transition function $\Delta$, one must associate a unique next state in $\mathrm{E}$ with each possible neighborhood state. Since there are $K=|E|$ choices of state to assign as the next state for each of the $\left|E^{N}\right|$ possible neighborhood states, there are $\mathrm{K}^{\left(\mathbf{K}^{N}\right)}$ possible transition functions $\Delta$ that can be defined.

\subsection{Computation in Cellular Automata}

Cellular automata may be viewed either as (1) computers themselves or as (2) logical universes, in which computers may be embedded.

In the first point of view, a cellular automaton is itself taken to be a computer. An initial configuration constitutes the data that the physical computer is working on, and the transition function implements the algorithm that is to be applied to the data. Every automaton is memory and processor at the same time. This is the approach taken in applications such as image processing or the Ising model. The Ising model is a simple model for a ferromagnet where every automaton at each lattice point $i$ computes the Hamiltonian (see below) for its two possible spin states, $s_{i}=1$ for spin up and $s_{i}=-1$ for spin down. In the Ising model only interactions between next nearest neighbor spins contribute to the Hamiltonian. The Hamiltonian is given by:

$$
H_{i}=-J \sum_{j \in N} s_{i} s_{j}
$$


where $J$ is the interaction energy between two neighboring spins and $N$ is the next nearest neighborhood template of spin $i$. In the stochastic Ising model the transition rules are non-deterministic. The Boltzmann distribution determines the probabilities for the two possible spin states at site $i$ :

$$
p_{i}(s)=\exp \left(-H_{i}(s) / k_{B} T\right)
$$

where $k_{B}$ is the Boltzmann factor and $T$ the temperature of the system.

The second view on computation claims that cellular automata may be viewed as logical universes in which a universal Turing machine, a machine which is capable of doing any computation if given the right programming, may be embedded. The initial configuration itself constitutes a computer, and the transition function is seen as the "physics" obeyed by the parts of this embedded computer. Both, the algorithm being run and the data being manipulated are functions of the precise state of the initial configuration of the embedded computer. The rules of the Game of Life support the embedding of a universal computer (see Chapter 4.3).

\subsection{Von Neumann's Work on Self-Replication}

John von Neumann was one of the first who recognized the second view of computational opportunities in cellular automata discussed above. Because of the importance of von Neumann's work on cellular automata and on the field of complexity in general, we give a short summary of his work. 
Besides his work in mathematics, development of digital computers, and quantum mechanics, von Neumann was interested in the ideas of self-replication. Von Neumann was convinced that the essence of self-replication in natural systems has an abstract logical form and that it is possible to implement it, at least theoretically, into an artificial system. Von Neumann found that a self-replicating machine needs to fulfill the two following requirements:

(i) the machine should be a universal constructor: given a description for any machine, it will search until it locates the proper parts, and then construct that machine. In particular, given a description of itself , it will construct a copy of itself.

(ii) the machine should contain a description copying machine capable of making a copy of the description of the universal constructor.

Put more formally, von Neumann said that the genetic material of any selfreplicating system, natural or artificial, has to play two fundamentally different roles. On the one hand, it has to serve as a program, a kind of algorithm that can be executed during the construction of the offspring. On the other hand, it has to serve as passive data, a description that can be duplicated and given to the offspring

In cellular automata John von Neumann found a system which was simple and abstract enough to be analyzed mathematically, yet rich enough to capture such a

\footnotetext{
${ }^{\mathrm{b}}$ A few years later, in 1953, Watson and Crick discovered that the genetic material, DNA, fulfilled von Neumann's two requirements precisely. DNA is a genetic program which encodes the instructions for making all the enzymes and structural proteins that the cell needs to function, and is a repository of genetic data which is duplicated and given to the new cell, at the same time.
} 
complex process as self-replication $^{c}[4,5]$. Von Neumann was able to prove that a selfreplicating pattern with about 200,000 cells could be implemented into a cellular automata with 29 different states ${ }^{d}$. These numbers show the immense complexity necessary to construct a self-replicating machine.

The result that cellular automata are capable of universal computation and even self-replication leads to several questions about cellular automata and complex systems in general. First, is it possible to classify cellular automata and systems in general and what are the characteristics of these different classes. Furthermore, which systems display complex behavior like universal computation and what causes this behavior. In Chapter 3 we will present some of the concepts and research results aimed at answering these questions.

\footnotetext{
${ }^{c}$ It was actually Stanislas Ulam, a Polish mathematician, who in the late 1940s suggested to von Neumann to implement his ideas about self-replicating machines in a programmable universe, which eventually became known as cellular automata.

d Von Neumann did not finish his studies on the theory of cellular automata before his death in 1954. Art Burks organized von Neumann's work, filled in remaining details and published the collection.
} 


\section{Chapter 3}

\section{Complexity and Criticality in Cellular Automata}

First, we present in this chapter Wolfram's and Langton's work on the classification of cellular automata and their theories about complexity in cellular automata. We then show how Langton made the connection between computation, complex cellular automata, and critical phenomena and discuss briefly characteristics of critical phenomena and self-organized criticality.

\subsection{Wolfram's Classification of Cellular Automata}

In 1984, Stephen Wolfram proposed an interesting classification for cellular automata [7]. He suggested that cellular automata can be classified like non-linear dynamical systems. Wolfram's contention was that all transition rules fall into one of four universality classes (see Fig. 2):

(i) Cellular automata rules in Class I lead to a very simple and static final state in a very short relaxation time, no matter what initial pattern the automaton started with. In dynamical systems, such rules correspond to a single point attractor in the phase diagram. 

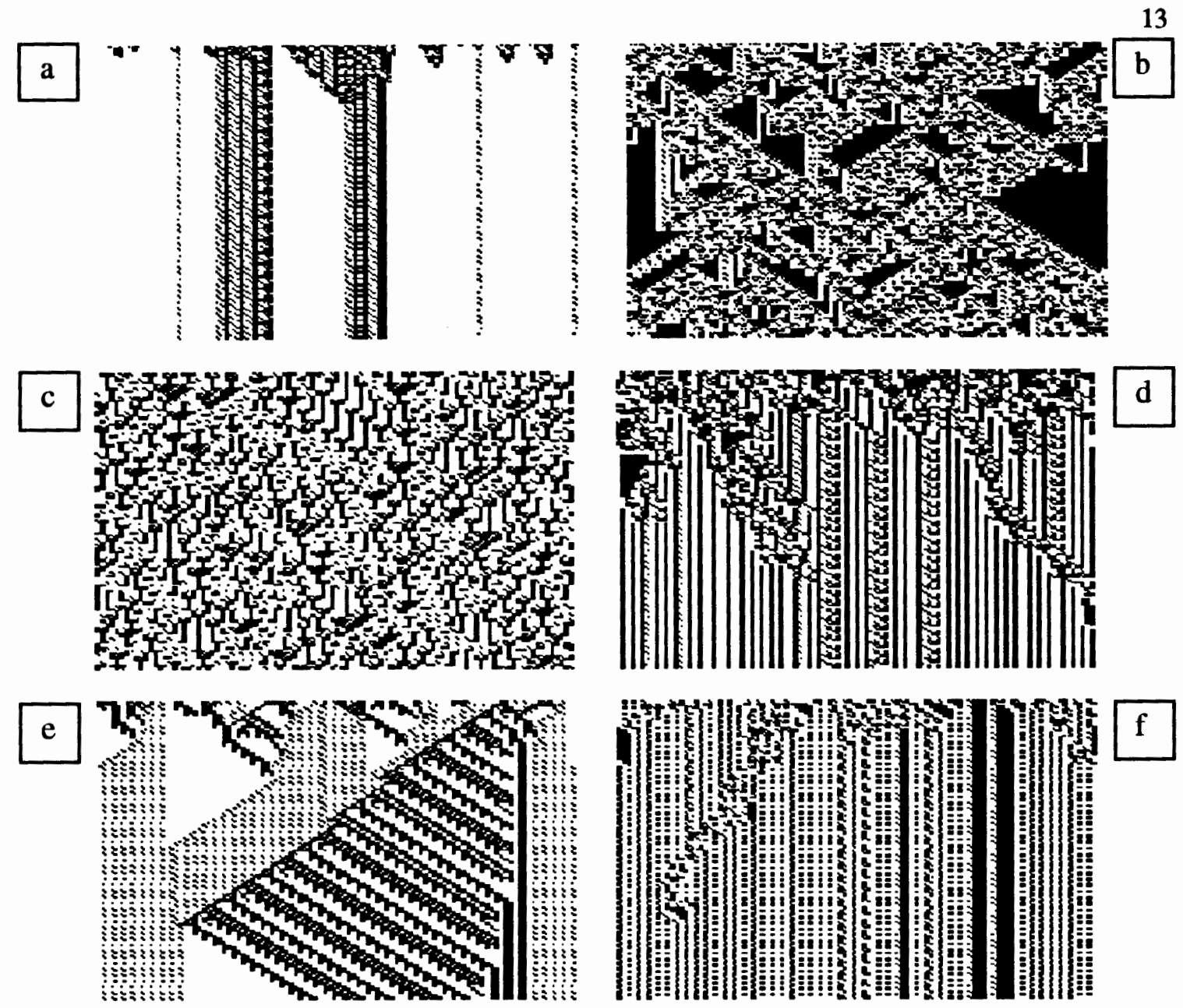

Fig. 2: This picture shows different time-space diagrams of one-dimensional, twostate cellular automata with one state shown black and the other white [6]. The initial configuration of the automaton is the first horizontal line. The lines below show the time evolution of the automata. Automata $a, d$, and $f$ belong to Wolfram's classes I and II. They show short lived transients and relax eventually into a static or periodic state. Automaton $c$ belongs to Class III. This automaton shows a chaotic dynamics, no static structures are visible. Automata $b$ and $e$ belong to Wolfram's Class IV automata. Both automata show static as well as propagating objects and exhibit long lived transients. 
(ii) In Class II rules, random initial conditions evolve into a set of static and oscillating patterns in a short relaxation time. In dynamical systems this behavior corresponds to periodic systems like a pendulum with periodic attractors in the phase diagram.

(iii) Wolfram's Class III rules display chaotic dynamics. A cellular automaton with this kind of rules never reaches a static or oscillating state. In Class III automata the activity is global and the whole automaton seems to boil. Wolfram connected this class of rules to chaotic dynamical systems which produce in the phase diagram socalled "strange attractors".

(iiii) Wolfram's Class IV rules do not correspond to any dynamical system and seem to be a special property of cellular automata. The behavior of Class IV rules is characterized by long-lived transients and the coexistence of static as well as propagating stable structures. The static and propagating structures are constantly interacting and lead to an ongoing complex dynamics that is far from equilibrium.

The appearance of propagating structures is the main difference of Class IV rules to Class II rules. The main difference to Class III rules is less activity which allows the existence of stable structures.

\subsection{Computation in Class IV Universes}

Of his four classes of behavior, Wolfram identifies Class IV as the class of complex cellular automata and the only class in which universal computation can take 
place. Wolfram claims that a universe capable of universal computation has to have three crucial properties:

(i) The universe needs to support information storage. Therefore a cellular automaton capable of universal computation needs to support static or periodic patterns for storing information.

(ii) The universe needs to support information transmission. Therefore a cellular automaton needs to support stable moving patterns which can transmit information. (For example in binary code: 1 if pattern arrives, 0 if pattern does not arrive.)

(iii) The universe needs to support information processing like reading and writing to the memory. Therefore a cellular automaton needs to support controlled creation and destruction of stable and moving patterns.

These three requirements for computation allow the implementation of universal computers just for cellular automata in Class IV ${ }^{\mathrm{a}}$. Class I/II rules cannot satisfy requirements (ii) and (iii) because they do not support propagating structures. Class III rules cannot satisfy requirement (i) because they are too active to sustain stable patterns for any length of time.

\footnotetext{
" The fact that cellular automata in Class IV can support universal computation leads to an interesting side effect of such cellular automata, the so called halting-problem. If a Class IV cellular automaton is programmed to solve a mathematical problem for which the result is not known, then it is unknown how the dynamics evolve and if the automaton will ever halt.
} 


\subsection{Langton's $\lambda$ parameter}

Wolfram did not make any claims on how one can decide in which class a given automaton with a certain state transition table would fall. The behavior of dynamical systems is often governed by a numerical parameter in the equation of motion of the system. For a dripping water faucet the parameter would be the rate of water flow. In the logistic map $x_{n+1}=a x_{n}\left(1-x_{n}\right)$, a simple model for population growth, the behavior of the function is determined by the parameter $a$. Both systems show for certain parameter values highly regular behavior like equal-sized drops and equal time spacing between drops, or a constant or oscillating population. But for other parameter values the system shows chaotic behavior.

Chris Langton suggested a similar parameter for cellular automata, called $\lambda$ $[8,9] . \lambda$ is defined as the probability that a cell will not be in the quiescent state in the next time step. The quiescent state is also called zero- or dead state. When a cell is in the quiescent state and all its interacting neighbors are in this state too, then the future state of the cell is again the quiescent state. Therefore, if all the cells on the lattice are in this state, the whole automaton will remain in this uniform "dead" state. The $\lambda$ parameter is defined formally in the following way: Pick an arbitrary state $s \in \mathrm{E}$, and call it the quiescent state $s_{q}$. Let there be $n$ transitions to this special quiescent state in a transition function $\Delta$. Let the remaining $\mathrm{K}^{\mathrm{N}}-n$ transitions in $\Delta$ be filled by picking randomly and uniformly over the other $\mathrm{K}-1$ states in $\mathrm{E}-s_{q}$. Then 


$$
\lambda=\frac{\mathrm{K}^{\mathrm{N}}-n}{\mathrm{~K}^{\mathrm{N}}} .
$$

If $n=K^{N}$, then all of the transitions in the rule table will be to the quiescent state $s_{q}$ and $\lambda=0.0$. If $n=0$, then there will be no transitions to $s_{q}$ and $\lambda=1.0$. When all states are represented equally in the rule table, then $\lambda=1.0-1 / \mathrm{K}$. The parameter values $\lambda=0.0$ and $\lambda=1.0-1 / \mathrm{K}$ represent the most homogeneous and the most heterogeneous rule tables, respectively. The behavior of all the Wolfram Classes should be captured between these two parameters. It turns out that $\lambda$ discriminates well between dynamical regimes for large values of $\mathrm{K}$ and $\mathrm{N}$, whereas $\lambda$ discriminates poorly for small values of $\mathrm{K}$ and $\mathrm{N}$.

Langton did his experiments on the dependence of the dynamics of cellular automata on the $\lambda$ parameter with one-dimensional cellular automata with 128 sites and periodic boundary conditions with $K=4$ and $N=5$ (two cells on the left and two cells on the right were included in the neighborhood template). Each array was started from a random initial configuration. The following regimes were found by Langton:

$0.0 \leq \lambda \leq 0.2$ : Class I behavior: a homogeneous fixed point in the quiescent state is reached after a few time steps.

$0.2 \leq \lambda \leq 0.45$ : Class I and Class II behavior: transient time is increasing strongly, automata show now homogeneous quiescent state or periodic objects. 
$0.45 \leq \lambda \leq 0.55$ : Class IV behavior: transient time is in the order of 12000 time steps, period times get much longer, moving patterns appear.

$0.55 \leq \lambda \leq 0.75$ : Class III behavior: no periodic behavior anymore, dynamics settles down to chaotic behavior.

Further important qualitative observations were made by Langton, which suggested that some of the behavioral characteristics of Class IV cellular automata is related to second order phase transitions in equilibrium thermodynamic systems:

- The transients grow rapidly in the vicinity of the transition between ordered and disordered dynamics, a phenomenon also known in the study of phase transitions as critical slowing down.

- The size of the array has an effect on the dynamics only for values of $\lambda$ in the Class IV region. The transient time does not depend on the array size up to $\lambda=0.45$. For $\lambda=0.50$ the transient time shows exponential dependence on the array size. For $\lambda=0.75$ the transient time does not depend anymore on the array size. This suggests that the correlations in Class IV are much bigger than in Class II and Class III and bigger than the size of the lattice with which Langton performed his simulations.

- The overall evolutionary pattern in time appears more random as $\lambda \rightarrow 0.75$. This observation can be confirmed by various entropy and correlation measures. $\lambda=0.75$ represents the state of maximal dynamical disorder. 
- the transition region supports both static and propagating structures. The propagating structures are like particles, quasi-periodic patterns of state change, which - like "gliders" in Conway's Game of Life - propagate through the array, constantly moving with respect to the fixed background of the lattice.

These observations led Langton to the conclusion that the Class IV behavior of cellular automata can qualitatively be compared with the behavior of systems at a second order phase transition, like the critical point in gas-liquid transitions, the demixing temperature of a binary fluid, or the Curie point of a magnet. $\lambda$ would then define the temperature scale in cellular automata.

Two complications show the limitations of the $\lambda$ parameter for characterizing the behavior of cellular automata. Langton used a so-called table-walk-through method to determine the rules for his automata. He started out with $\lambda=0.00$, where all transition rules lead to the quiescent state, and assigned then continuously and randomly other states than the quiescent state to the transition rules. With this method the transition points for different experimental runs were not always at a specific critical value $\lambda_{c}$ but spread out over a bigger regime. The second problem was that not all of his experiments showed a second order phase transition. Often the cellular automata jumped directly from ordered to chaotic behavior which is characteristic for first order phase transitions. 


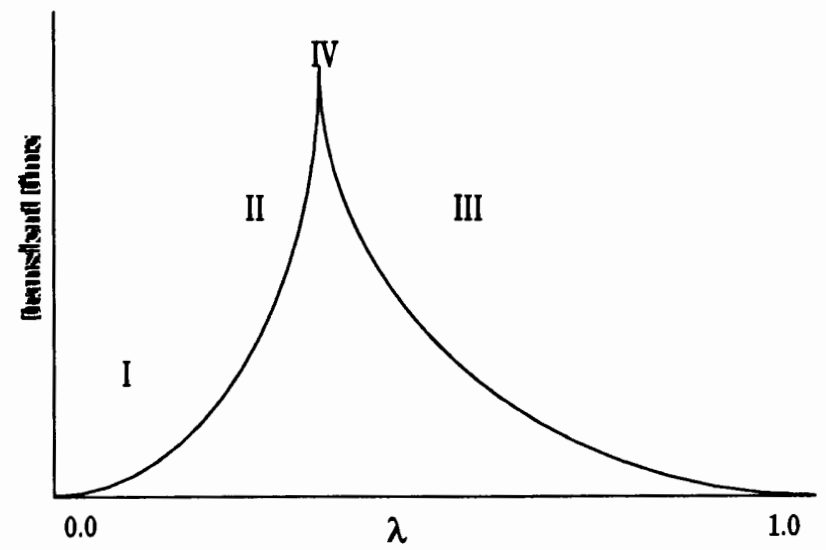

Fig. 3: Langton's results for transient time vs. $\lambda$ and Wolfram's classification of cellular automata.

\subsection{Continuous Phase Transitions}

Because Langton claims that cellular automata show characteristics of a second-order or continuous phase transition we will briefly summarize this topic in the following paragraphs.

A phase transition is signaled by a singularity in a thermodynamic potential. If there is a finite discontinuity in one or more of the first derivatives of the appropriate thermodynamic potential the transition is called first-order. For a magnetic system the free energy $F=U-T S$, where $U$ is the total energy, $T$ the temperature, and $S$ the 
entropy of the system, is the appropriate potential. If the first derivatives are continuous but second derivatives are discontinuous or infinite the transition will be described as higher order, continuous, or critical. This type of transition corresponds to a divergent susceptibility, an infinite correlation length, and a power law decay of correlations.

A central role in the theory of critical phase transitions is played by the critical exponents. The critical exponents define the divergence of thermodynamic quantities at the critical point where the continuous phase transition occur.

Let

$$
t=\frac{\left(T-T_{c}\right)}{T_{c}}
$$

be a measure of the deviation in temperature from the critical temperature $T_{c}$. Then the critical exponent associated with a function $F(t)$ is

$$
\varepsilon=\lim _{t \rightarrow 0} \frac{\log |F(t)|}{\log |t|}
$$

or, as it is more usually written,

$$
F(t) \sim|t|^{\varepsilon}
$$

Equation (3.4) represents only the asymptotic behavior of the function $F(t)$ as $t \rightarrow 0$. Table 1 shows the most common thermodynamic quantities for a magnetic system and the asymptotic behavior of these quantities at the critical point. 

Zero-field specific heat
$C_{H} \sim|t|^{-\alpha}$
Zero-field magnetization
$M \sim(-t)^{\beta}$
Zero-field isothermal susceptibility
$\chi_{T}-|t|^{-\gamma}$
Critical isotherm $(\mathrm{t}=0)$
$H \sim|M|^{\delta} \operatorname{sgn}(M)$
Correlation length
$\xi \sim|t|^{-v}$
Pair correlation function at $T_{c}$
$G(\vec{r}) \sim 1 / r^{d-2+\eta}$

Table 1: Definitions of the most commonly used critical exponents for a magnetic system.

It is interesting to note that the critical temperature $T_{c}$ is dependent on the interatomic interactions of the system but the critical exponents are to a large degree "universal" depending only on a few fundamental parameters. For models with shortrange interactions these are the dimensionality of space, $d$, and the symmetry of the order parameter. For example different physical systems like uniaxial ferromagnets, fluids near a critical point, mixtures of liquids near consolute points, and alloys near order-disorder transitions all have the same critical exponents and belong therefore in the same universality class. A theoretical model for all these physical systems is the three dimensional Ising model. 


\subsection{Correlation Functions}

Thermodynamical quantities like the specific heat $C_{H}$, magnetization $M$, or susceptibility $\chi$ are macroscopic quantities. A fuller understanding of phase transitions and critical phenomena can be obtained by considering what is happening on a microscopic level. Correlation functions have been introduced to do this in a quantitative way. For example the spin-spin correlation function, defined to measure the correlation between the spins on sites $i$ and $j$, is

$$
\Gamma\left(\vec{r}_{i}, \vec{r}_{j}\right)=\frac{1}{N^{2}} \sum_{i, j}\left(s_{i}-\left\langle s_{i}\right\rangle\right)\left(s_{j}-\left\langle s_{j}\right\rangle\right)
$$

where $N$ is the total number of spins, $\vec{r}_{i}$ the position vector of site $i$ and $\langle E q n .(3.5)\rangle$ the average taken over the whole system. If the system is translational invariant, $\left\langle s_{i}\right\rangle=\left\langle s_{j}\right\rangle$, and $\Gamma$ depends only on $\left(\vec{r}_{i}-\vec{r}_{j}\right)$, then

$$
\Gamma\left(\vec{r}_{i}-\vec{r}_{j}\right) \equiv \Gamma_{i j}=\left\langle s_{i} s_{j}\right\rangle-\langle s\rangle^{2}
$$

Away from the critical point the spins become uncorrelated as $r \rightarrow \infty$ and hence the correlation function decays to zero. This is true not only above but also below the critical temperature, although here the mean value of the spin $\langle s\rangle \neq 0$, because, according to Eqn. (3.5), the correlations are measured between fluctuations of the spins away from their mean values. The correlations decay to zero exponentially with the distance between the spins 


$$
\Gamma(r) \sim r^{-\tau} \exp \left(\frac{-r}{\xi}\right)
$$

Equation (3.7) provides a definition of the correlation length, $\xi$, which can be used as an estimate of the size of the largest fluctuation, for example the largest cluster of connected up or down spins in an Ising model.

At the critical point these fluctuations become infinite, one cluster can extend over the whole system, and Equation (3.7) breaks down. Evidence from experiment and exactly soluble models shows that here the correlation function decays as a power law

$$
\Gamma(r) \sim \frac{1}{r^{d-2+\eta}}
$$

This means that at the critical point fluctuations on all length scales occur and the spatial structure of the system is self-similar or fractal.

The correlation function can be related to macroscopic thermodynamic quantities. For example the spin-spin correlation function in ferromagnetic systems can be related to the fluctuations in the magnetization and hence to the susceptibility. The fluctuations of the magnetization are given by:

$$
\left\langle(M-\langle M\rangle)^{2}\right\rangle=\left\langle M^{2}\right\rangle-\langle M\rangle^{2}=k^{2} T^{2} \frac{\partial^{2}}{\partial H^{2}} \ln Z=k T \chi_{T}
$$

where $Z$ is the partition function of the system. If the magnetization is written as a sum over spins, 


$$
\left\langle(M-\langle M\rangle)^{2}\right\rangle=\sum_{i}\left(s_{i}-\left\langle s_{i}\right\rangle\right) \sum_{j}\left(s_{j}-\left\langle s_{j}\right\rangle\right)=\sum_{i j} \Gamma_{i j}
$$

For a translational invariant system

$$
\sum_{i j} \Gamma_{i j}=N \sum_{i} \Gamma_{i 0} \sim N \int \Gamma(r) r^{d-1} d r
$$

where the sum has been replaced by an integral, a step justified near criticality where the correlation length is big and the lattice structure gets unimportant. Combining Eqn. (3.9), (3.10), and (3.11) leads to

$$
\chi_{T} \sim N \int \Gamma(r) r^{d-1} d r
$$

At the critical temperature the susceptibility diverges and hence $\Gamma(r)$ must become sufficiently long range that the integral on the right hand side of Eqn. (3.12) diverges. This sets an upper limit on $\eta$ of 2. From (3.9) it follows that a divergent susceptibility also implies a divergence in the fluctuations of the magnetization.

\subsection{Self-Organized Criticality}

We presented in section 3.3 and 3.4 the characteristics of continuous or critical phase transitions. In this section another concept, called self-organized criticality, will be introduced which aims to explain a different kind of critical phenomena in certain systems. Self-organized criticality has also been reported for the Game of Life.

P. Bak, C. Tang and K. Wiesenfeld proposed a few years ago a concept called self-organized criticality $[10,11]$. Self-organized criticality tries to explain and connect 
two characteristic phenomena of certain extended dissipative dynamical systems, the so-called $1 / f$ or "flicker" noise and spatial fractal structures. $1 / f^{\alpha}$ noise power spectra have been reported for many different kind of physical systems like the light intensity of quasars, the sunspot activity, the current through resistors, water flow in rivers, sand flow in hour glasses and others. It has also been pointed out that nature is full of self-similar fractal structures [12]. The common feature for all these systems is that the power-law temporal or spatial correlations extend over a wide scale range. This suggests that the mechanism causing the events are the same on all scales although one would expect that the physics describing events on different scales would vary. Self-organized criticality suggests that the power-law behavior in time and space in extended dissipative dynamical systems is the result of the critical state in which these systems organize themselves. This organization occurs without the fine tuning of a parameter like the temperature in the case of magnetic or gas-fluid continuous phase transitions.

The concept of self-organized criticality in some way complements the concept of chaos where simple systems with a small number of degrees of freedom display quite complex behavior and long range correlations.

The standard model for self-organized criticality is a sand pile model, introduced by Bak, Tang, and Wiesenfeld, and therefore also called the BTW model [10]. One version of this model was implemented on a cellular automaton and examined numerically. In two dimensions the cell state $z$ was updated as follows: 


$$
\begin{aligned}
& z(x, y) \rightarrow z(x, y)-4, \\
& z(x \pm 1, y)=z(x \pm 1, y)+1 \\
& z(x, y \pm 1) \rightarrow z(x, y \pm 1)+1
\end{aligned}
$$

if $\mathrm{z}$ exceeds a critical value $\mathrm{K}$. There are no parameters since a shift in $\mathrm{K}$ simply shifts z. The cellular variable may be thought of as the local slope of the sand pile in some direction. In the original paper the system was set up with random initial conditions $z \gg K$, and was then simply allowed to evolve until the dynamics stopped, i.e., $z \leq K$ for all z's. The dynamics was then probed by measurements of the response of the resulting state to small local random perturbations. The perturbations caused avalanches on all size scales, distributed on a power-law, and only limited by the size of the system. The same results were found when the initial state was sub-critical and the system was slowly driven by injecting quantized energy in the form of a "sand corn" at a random position. The sand-pile would grow until it reaches a critical size, depending on the critical slope, where avalanches on all size scales occur. To support avalanches on all size scales, similar to fluctuations in a ferromagnet at the critical point, the correlations in the system have to be larger then the system size.

Probably the most interesting aspect of self-organized criticality is that it has not just been reported for conservative models like the sand pile but also for nonconservative models $[13,14,15,16]$. This finding led to the claim that self-organized criticality could constitute the physical principle behind many natural phenomena, even phenomena in living systems. Raup has argued that biological evolution is in fact 
intermittent rather than gradual [17]. Periods of stasis are interrupted by events where many species become extinct. Bak reported that the distribution of the magnitudes of those events seems to be power-law like. Bak claims that this can be taken as an indication that biology operates at a self-organized critical point, in which case no external cataclysmic force is necessary to bring about major disasters such as the extinction of the dinosaurs. Kauffmann and Johnson have studied models of evolving interacting species, and found evidence of self-organized criticality [18]. Moreover, Ray has performed simulations of life evolving in the memory of a computer, and also found intermittent events with power-law distributions. All these results have been put together in the idea called "evolution to the edge of chaos", emphasizing that many natural systems have evolved or organized themselves to an intermittent state between a disordered, chaotic and a frozen, ordered state.

These results, found for natural systems, imply that complexity and criticality are somehow related in systems. Complexity may even cause criticality. The idea of complex systems being at "the edge of chaos", between a chaotic and an ordered state, has also been used by Langton to describe his results for cellular automata. These similarities indicate that there are general principles underlying complexity in such different systems as natural systems and cellular automata. Self-organized criticality could be such a general principle. 


\section{Chapter 4}

\section{The Cellular Automaton Game of Life}

We introduce in this chapter the cellular automaton Game of Life. We present the most important results which have so far been reported for Life. We discuss why Life is considered to be a complex cellular automaton and show why this question is important for the theory of complex systems in general.

\subsection{Introduction}

Conway's Game of Life is probably the most famous cellular automaton $[3,19]$. It has been claimed that Life captures, in an allegorical sense, some of the complex features of real biological life [20]. It is Life's complexity, originating from very simple rules, which makes it so fascinating.

Life is a two state cellular automaton defined on an $L \times L$ square lattice of sites. In each time step the fate of each site is dependent on its eight nearest neighbors and parallel updating according to the following rules:

(i) A cell in the alive state will stay alive in the next generation if it has two or three alive neighbors, otherwise it will be dead. 
(ii) A cell in the dead or empty state will be in the alive state in the next generation if it has exactly three alive neighbors (birth), otherwise it will stay in the empty state.

If started with random initial conditions, Life generates and destroys a multitude of different objects during the evolution process until it reaches the final steady state. The final steady state consists mostly of a population of five different objects. These objects are commonly known as the oscillatory blinker and the still-lifes block, boat, beehive, and burloaf. These objects are shown in Fig. 4. Objects with a probability of less then $2 \%$ and objects like the traffic light, built of four blinkers, or the honey farm, built of four beehives, are not shown.

Probably the most interesting object in Life is the glider, a periodic and moving pattern. The glider is an object with period four, which means that the glider reappears in its original shape after 4 time steps. But the glider also moves one cell diagonally from its former position. The four phases of the glider are shown in the second row of Fig. 4. A further, very important discovery in Life was the glider gun, an object with period 30 which ejects a single glider every cycle. The glider gun was essential for Conway's proof that Life is capable of universal computation (see Chapter 4.3). 

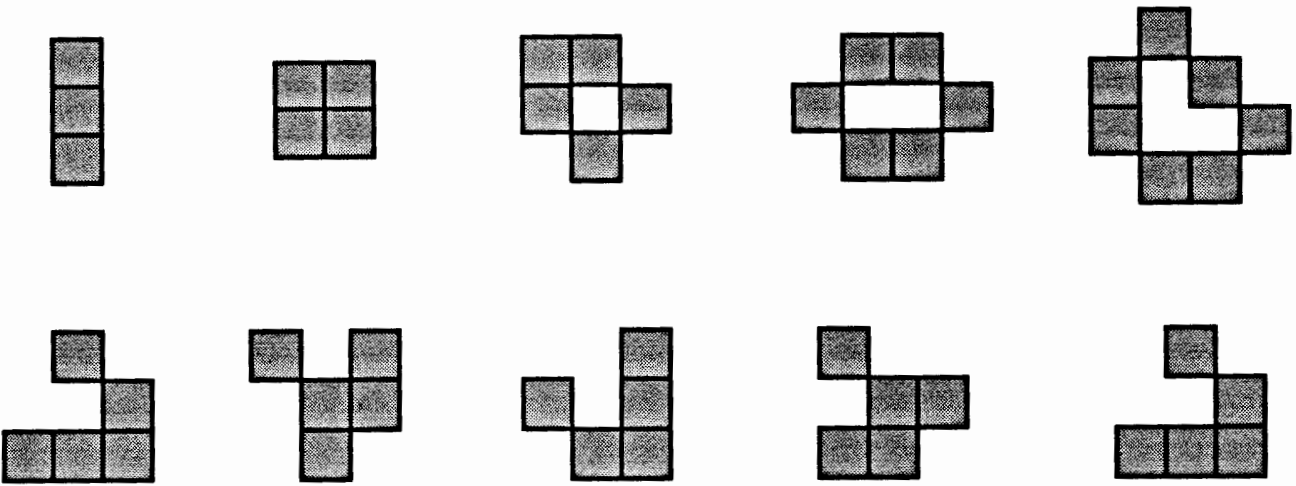

time 0

time 1

time 2

time 3

time 4

Fig. 4 The first row shows the five objects in the final steady state of Life. The probability for their appearance in the final steady state are from the right to the left: blinker 35\%, block 33\%, boat 6\%, beehive 20\%, burloaf 6\%. The second row shows the four different phases of the glider.

The most interesting question about Life is whether or not its dynamics is critical. Life is a very complex cellular automata, and we will show in Chapter 4.3 that the Life universe even supports universal computation. Because of properties like long-lived transients, propagating structures, universal computation, Life clearly belongs to Wolfram's Class IV cellular automata. Langton claims that cellular automata in Class IV display dynamical behavior similar to thermodynamic systems at the critical point (see Chapter 3.3); therefore Life should also display critical behavior. Bak et. al. [15] claims that they have found self-organized criticality in the Game of Life (see Chapter 4.4) and other models of complex systems, as well as natural systems 
(see Chapter 3.6) and that self-organized criticality could therefore be a universal concept for explaining very different kinds of complex systems. But the result that the Game of Life is a self-organized critical system has been questioned [22] and the question is not yet resolved [14, 23].

\subsection{Dynamical Properties of Life}

Life has many interesting dynamical properties besides the ability of universal computation (see Chapter 4.3). In fact Langton's and Wolfram's work suggests that cellular automata capable of computation require an "interesting" dynamics (see Chapter 3.1, 3.2).

The best way to understand how Life got its name is to watch Life's evolution on a computer screen with each of the two states corresponding to a different color. If Life is started with a random population of alive cells then it depends on the initial density of alive cells, $\rho_{0}$, how Life's dynamics will evolve.

Three different regimes have to be distinguished. In regime I, for $\rho_{0}<0.1$, the initial alive cells are so sparse that most of them die in the first few time steps because of "under population". Life quickly relaxes in regime $I$ into its final steady state, populated with the objects shown in Fig. 4. The fate for the Life universe is very similar in regime III, for $\rho_{0}>0.7$. But here the cause for the fast decay of activity and alive sites is "over population". Most cells which are alive in the initial time step die in 
the first time step and then the population of alive cells is too sparse to sustain activity for a longer period of time. In regime I and III, the active regions are few and sparse and therefore interactions rarely happen. The final density of alive cells, $\rho_{\infty}$, depends on the initial density $\rho_{0}$ in these two regimes [24].

In regime II, $0.15 \leq \rho_{0} \leq 0.7$, the final density of alive cells is a constant, $\rho_{\infty}=0.0285[24]$, and does not depend on $\rho_{0}$. In regime II, Life shows the most interesting and diverse dynamical behavior:

For time $t<30$ the computer screen displaying the dynamics looks like a chaotic boiling soup. After this initial period, when the correlations to the initial configuration have been destroyed, the dynamics of Life is independent from the initial configuration. In the second time period, called active period, Life displays clusters of activity separated by different sized areas of inactivity. The inactive areas are populated by the same static and periodic objects as the final steady state. Outbursts of the activity regions occur and activity clusters merge. Bagnoli et. al. [24] reported that the number of alive cells decays like a power-law with time in the active period, suggesting the dominance of correlations (see Fig. 5). Bagnoli et. al. also reported that the active period characterizes the behavior for $30<t<t_{c}$ with $t_{c} \sim 2000$ being not lattice size dependent, which suggests that the correlations in the active period are not 
bigger than the lattice sizes used in the simulations ${ }^{a}$. Figure 5 shows that the powerlaw decay for alive cells ends when the density drops below $\rho_{c} \approx 0.04$.

The last time period, $t>t_{c}$, is called glider period. Here clusters of activity are few and separated by vast areas of non-activity, populated by still and periodic objects. In this period, information can only be transmitted with gliders. If Bak's claim is correct that the final steady state of Life is critical, then the glider period would go on forever for infinite lattice sizes (see Chapter 4.4). This conclusion fits with the observation of Bagnoli that there seems to be no time scale in the glider regime. No rule, like the power-law decay for alive cells in the active period, seems to govern the glider period, and no prediction can be made when the automaton will settle down into the final steady state.

\subsection{Universal Computation in Life}

Conway not only invented Life but also showed that Life is capable of universal computation, even capable of von Neumann's idea of universal construction $[19,25]$ (see Chapter 2.3) $)^{b}$. As was pointed out in Chapter 3.2, computation in a cellular automaton requires processes which allow storage of information, transmission of information, and writing as well as reading of information. For the transmission,

a Bagnoli et. al. measured $t_{c}$ for $L>250$. Our simulations showed that $t_{c}$ is lattice size dependent for $L<100$. $\rho(t)$ varies much more for smaller $L$ but we also found the power-law decay for alive cells in the active period.

'The earlier discovery of the glider gun was crucial for the finding that Life is capable of universal computation and universal construction. The glider gun was discovered when Conway offered a $\$ 50$ prize for the person who first proves or disproves that Life can support patterns which grow forever. 


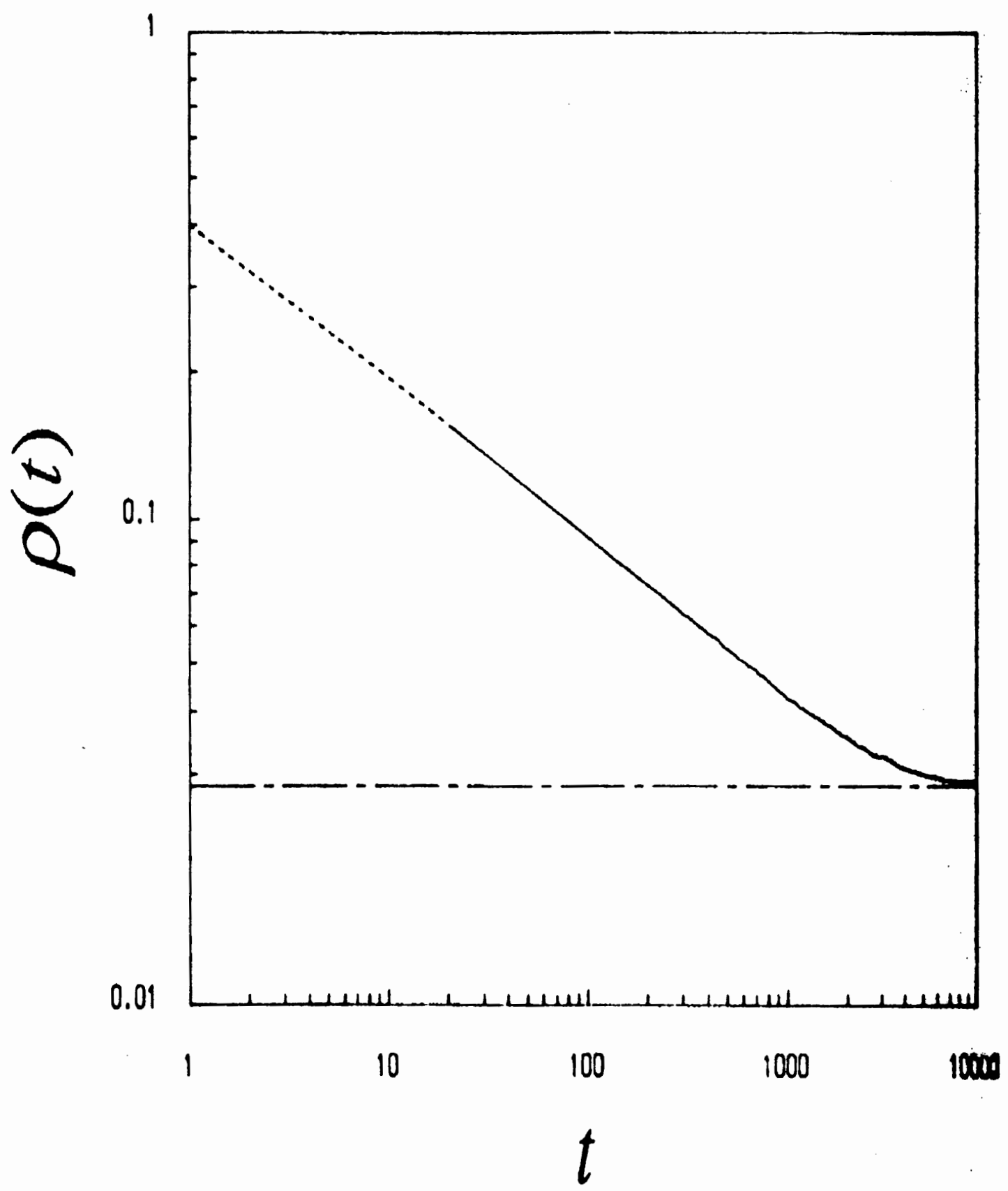

Fig. 5: This figure shows the results reported by Bagnoli et.al. for the time evolution of the alive site density $\rho(t)$ in Life. The active period is characterized by the power-law decay. The power-law decay ends at about $t_{c} \approx 2000$ and $\rho\left(t_{c}\right) \approx 0.04$, where the glider period starts. 
writing and reading of information, the cellular automaton needs to support moving and stable objects like the glider. In binary code, a glider at a certain place at a certain time can be interpreted as 1 and no glider as a 0 . Streams of gliders, generated by glider guns, have then the same function as wires in a "real" computer - they transmit bits of information. When gliders collide controlled with each other or with static objects, information is either altered or processed.

Conway showed that it is possible to implement the three fundamental logic gates, AND, OR, and NOT, into Life, and that therefore universal computation is possible. The inputs of these logic gates are thinned and coded glider streams. In Conway's construction of the three fundamental logic gates uncoded glider streams are also needed. The glider streams are timed and spaced such that certain gliders collide and vanish. A further object, the eater, is required as well for computing in Life. The eater is able to destroy unneeded gliders without being destroyed itself.

Conway showed further that the block can be used as a storage medium. The distance of the block from a certain point serves as the coded information. Flotillas of gliders are able to move the block in any direction to increase or decrease the distance of the block. These processes can be used to write or read the information stored in the distance of the block.

As mentioned at the beginning of this chapter, Life is not just capable of universal computation but also of universal construction. This task is even more complex and can be accomplished only because flotillas of gliders can construct all the 
necessary objects for a universal computer in the Life universe: the block, the eater, and the glider gun.

\subsection{Self-Organized Criticality in Life}

As mentioned before, self-organized criticality has been reported for the Game of Life [14, 15]. But the discussion about this finding is not yet resolved [22].

Bak et. al. claimed that if the final steady state of Life is perturbed similar to the sand-pile model, for example by adding an alive cell at a random location, then Life shows spatial and temporal activity distributed on a power-law. It is not totally clear how Bak defines activity. We define active sites as sites which have changed their state from the past time step, but we exclude periodic active sites. We argue that periodic activity, in Life mainly the blinker, is not correlated to active sites far away. The active sites of the period-two blinker are only influenced by their next nearest neighbors. Bak did not mention the exclusion of periodic activity in his simulations. Because the periodic blinker is the most common object in the glider regime (see Fig.4), this would definitely influence and distort Bak's results.

\footnotetext{
'This led Conway and others to the idea that the Life universe could evolve a whole ecosystem, similar to our natural world. The idea is that if the Life universe would be made big enough, and several self-replicating Life machines were implemented into this universe, replicates would be mutated because of disturbances from other objects and the self-replicating machines could start to evolve. Therefore an evolving ecosystem with competition for space and other resources is thinkable in the two dimensional discrete Life world.
} 
The spatial activity, the size $s$ of a avalanche, was defined by Bak as the total sum over time and space of births and deaths, following a single perturbation. Bak et. al. found for the distribution $D(s)$ of avalanches with size $s$ :

$$
D(s) \sim s^{-\tau}
$$

with $\tau \approx 1.4$. Bak's definition of "avalanche" activity is not explained clearly and seems somehow arbitrary. He emphasizes similarities of the activity in Life to the sandpile model. But it remains unclear why Bak's definition of spatial activity resembles more similarity to the sand-pile model than other definitions. We think that the sum of births and deaths over space only would be more similar to the sand-pile model than Bak's definition. These definition problems arise because Life is a non-conservative model.

A power-law distribution was also reported for the temporal distribution $D(T)$ of the activity. The life time of a perturbation was defined by Bak as the total number of time steps it takes for Life to relax again into a steady state after a perturbation. The reported result is:

$$
D(T) \sim T^{-b}
$$

with $b \approx 1.6$. 

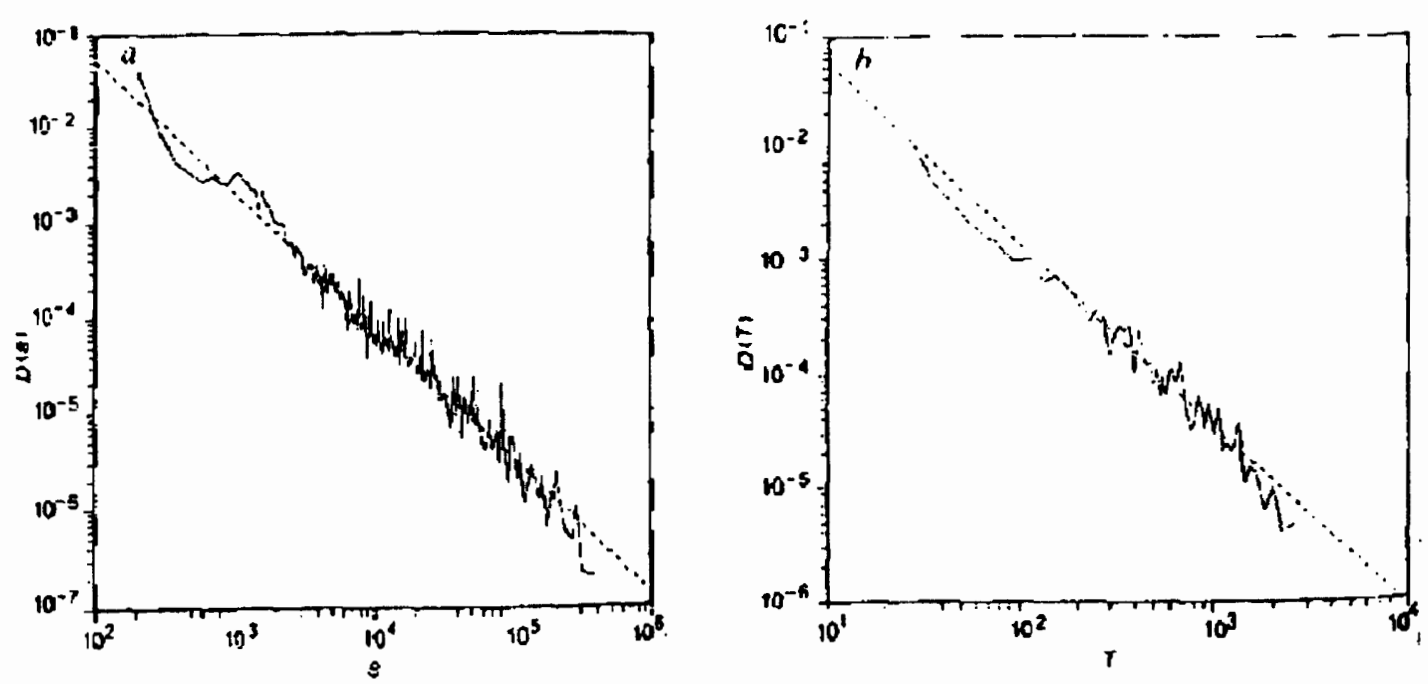

Fig. 6: Size distribution of avalanches $D(s)$ and relaxation time distribution $D(T)$ after a perturbation of the final steady state of Life, reported by Bak et al.

Bak concludes from the fact that the activity does not decay or explode exponentially that life and death are highly correlated in time and space: the system has evolved into a critical state. This conclusion is also supported by another result reported by Bak, namely that the activity in Life is sustained on a fractal. Bak reported that the number distribution $D(r)$ of active sites at a distance $r$ in an active cluster increases with $r$ as

$$
D(r) \sim r^{D-1}
$$

with the fractal dimension $D \approx 1.7$. 


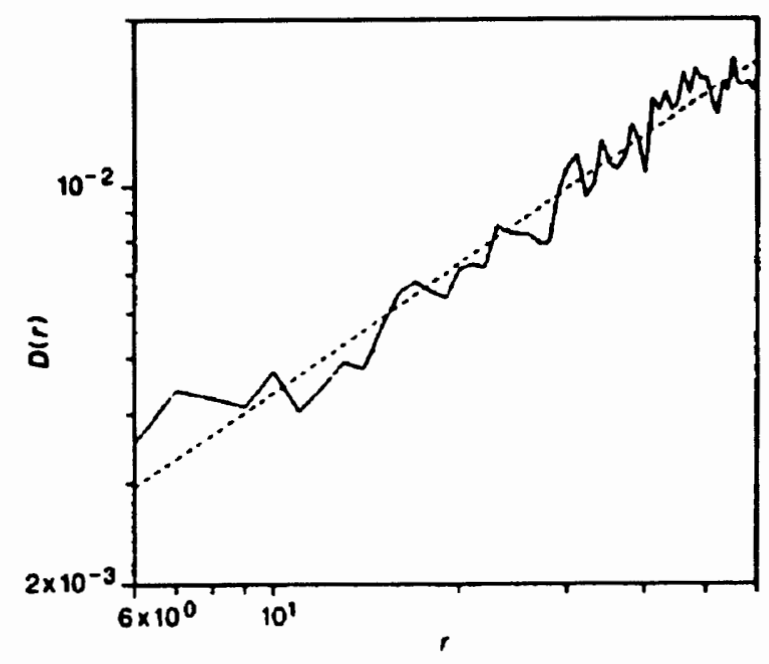

Fig. 7: Number distribution of active sites $D(r)$ vs. distance $r$ after perturbations of final steady state, reported by Bak et. al[24].

Bennet $e t . a l$. reported results which contradict self-organized criticality in Life[22]. The power-law distribution for the equilibration time implies that the average equilibration time is infinite for infinite lattice sizes. Bennet $e t$. al. reported that the average equilibration time approaches a constant for lattices larger than $L=100$. This suggests a characteristic extinction length and therefore a finite correlation length. But these results have been challenged too and the question if Life is critical or not have not yet been resolved.

Whether or not Life is critical is important in several ways. First, Life is a Class IV cellular automaton with all the characteristics of an extremely complex system. If Langton's result is correct that cellular automata undergo a second-order phase transition at the same location in rule space where complexity occurs, then one would 
expect that these Class IV cellular automata show critical behavior. Further, if it could be shown that the non-conservative Game of Life is a self-organized critical system then this would support the claim that other non-conservative systems, like natural systems, could posses this property as well. There have been doubts if systems without a conservation law can exhibit correlations over large scales [26]. Bak claims that selforganized criticality could even be a general concept for explaining complexity in natural systems as well as in artificial systems. 


\section{Chapter 5}

\section{Results and Discussion}

We report in this chapter several new results about Life. We investigate the box count dimensionality and report two different scale regimes, a small scale regime with a fractal dimensionality and a large scale regime with dimensionality two. We also examine the correlation between alive cells and find that the correlations extend farthest in the active period and decrease in the glider period. Further, we report findings about the dependence of correlations on the spatial direction in the Life universe. Last, we examine the activity cluster size distribution in the active and glider period. Our results do not support Bak's claim that activity in Life is sustained on a fractal.

\subsection{General Methodology used in Simulations}

All of the simulations for this thesis are programmed in Visual Basic for DOS and run on a PC $486 \mathrm{DX} 2$ with $66 \mathrm{MHz}$. Different lattice sizes $L$ with $78 \leq L \leq 256$ and periodic boundary conditions are used. The initial state of each simulation contains a random population of alive cells with an initial density of $0.2 \leq \rho_{0} \leq 0.4$. The simulation is stopped when the activity in the Game of Life is limited to cell state changes of period two. Periodicity higher than two does not occur in any of our simulations. 


\subsection{Distribution of Objects in the Final Steady State of Life}

The reported distribution of objects in the final steady state of Life (see Fig 4) results from the average of ten runs for each of the following lattice sizes $L: 78,99$, 120 and 160. Objects like the honey farm or the traffic light, consisting of four beehives or four blinkers respectively, are not counted as separate objects. Objects occurring with a probability of less then $1 \%$ are also not counted. The following probabilities are found for the different objects: blinker $35 \%$, block $33 \%$, boat $6 \%$, beehive $20 \%$, and burloaf $6 \%$.

\subsection{Fractal Box Count Dimensionality of the Final Steady State of Life}

The fractal dimensionality of the final steady state of Life is examined to distinguish different spatial scale regimes. Garcia et. al. suggested that the final steady state of Life is a set of randomly distributed objects [27]. They examined how the population of alive sites, $M(r)$, increases with an area of radius $r$. They found the following power-law relationship:

$$
M(r) \sim r^{D_{G}}
$$


with $D_{G}=1.94 \pm 0.06$. Garcia et.al. stated that this result is not conclusive. Garcia et.al. did not distinguish between different scale regimes. If Life consists of randomly distributed objects we expect $D_{G}=2$ only for large $r$.

\subsubsection{Box Count Method}

The box dimension or box counting dimension $D$ of a set is defined in the following way:

$$
N(\delta) \sim \frac{1}{\delta^{D}}
$$

$N(\delta)$ is the number of boxes needed to cover the set as a function of the boxsize $\delta$. The box count method was introduced by Benoit Mandelbrot and others to determine the fractal dimensionality of objects [12]. A fractal dimension is caused by the self-similarity of the fractal object. Self-similar objects do not have a characteristic length scale in the scale range where the fractal dimensionality is valid. If, for example, a measured coastline has a well defined length $L$ we expect $L(\delta)=N(\delta) \delta$ to approach this value for $\delta \rightarrow 0$. This is not the case for many coastlines. In fact, the measured length $L(\delta)$ often diverges and is nicely approximated by the following power-law relationship:

$$
L(\delta)=a \delta^{1-D}
$$

For an ordinary, Euclidean curve we would expect $a$ to be $L$ and at least for small enough $\delta$ the exponent D should be equal to one. We find, however, that for example 
the coastline of Britain is a fractal with a fractal dimension $D \cong 1.3$. The coastline of Norway was determined to be $\mathrm{D} \cong 1.52$ [28]. The fractal dimensionality for a coastline has to be between one and two - the integer dimensionalities of a line and an area. The larger fractal dimensionality for the coastline of Norway indicates that Norway's coastline is rougher and more "area-filling" then Britain's coastline. This difference can easily be seen on maps.

We expect for the Game of Life different dimensionalities for different scale regimes. We expect for box sizes smaller than the average distance between neighbor objects a fractal dimensionality between zero and one.. A disconnected set of points has a box count dimensionality of zero for box sizes smaller than the shortest distance between two points. The number of occupied boxes is in this scale range not dependent on the box size, therefore the box count dimensionality has to be zero (see Eqn. (5.2)). The individual cells represent the points for the box count method in Life. Because the objects in the final steady state of Life consist of groups of connected cells, but the objects themselves are disconnected, a box count dimensionality between zero and one is expected. If the objects are randomly distributed, we expect a dimensionality of 2 for box sizes larger than the average distance between next nearest objects. 


\subsubsection{Theoretical Models for the Fractal Box Count Dimensionality in}

\section{Life}

$N(\delta)$ in Eqn. (5.2) represents for Life the number of boxes with box size $\delta$, needed to cover the set of alive cells. Therefore, each counted box contains at least one alive cell. The density of alive cells in the final steady state is a universal property of Life (see Chapter 4.2). Therefore, a rescaled number of occupied boxes is introduced:

$$
\rho(\delta)=\frac{N(\delta)}{L^{2}}
$$

Note that $\rho(1)$ is equal to the density of alive cells.

\section{A. Large Scale Regime}

A randomly distributed set in two dimensions has a box count dimensionality of two for box sizes larger than the average distance between next nearest objects. We show this, for reasons of simplicity, only with randomly distributed cells on a square lattice of size $L$. The probability that a box of size $\delta$ does not contain a cell is given by:

$$
p_{\delta}=\left(1-p_{0}\right)^{\delta^{2}}
$$

where $p_{0}$ is the probability for a site to be occupied by a cell. $1-p_{\delta}$ is then the probability that a box of size $\delta$ does contain any cell. Therefore the average number of boxes of size $\delta$ which contain at least one cell is: 


$$
N(\delta)=\left(1-\left(1-p_{0}\right)^{\delta^{2}}\right) \frac{L^{2}}{\delta^{2}}
$$

which yields for $\delta>>1$

$$
N(\delta) \cong \frac{L^{2}}{\delta^{2}}
$$

It follows for the dimensionality with Eqn. (5.2) that $D_{l}=2$ for $\delta>>1$. We can conclude that we will find a dimensionality of two for Life if the objects are randomly distributed.

\section{B. Small Scale Regime}

The alive cells in the final steady state of Life are obviously not randomly distributed but rather organized in objects. Therefore, a box count dimension of two is not expected for box sizes smaller than the average distance between objects.

The rules of the Game of Life lead to the phenomenon that in the final steady state almost all objects are separated by at least two empty cells. The transition rules of Life determine that clusters of alive cells, like the five common objects in the final steady state, can only be static if all alive cells have either two or three alive neighbors, and the neighboring empty cells have either less than three or more than three alive cells. Cells in Life are interacting with their eight surrounding neighbors; therefore all stable configurations stay stable if separated by at least two empty cells from other configurations. There are many constraints for possible stable configurations of two objects either touching or separated by just one cell. It is possible that certain 
configurations of close objects do not occur although they are theoretically stable because there exist no, or few predecessors to this final stable configurations ${ }^{a}$.

The most common violation of the two cell excluded area assumption occurs with objects like the traffic light or the fleet, built out of four beehives. These stable configurations, although built up of objects from the five common ones, should actually be considered objects on their own. They have their own specific predecessors and their own evolution. Like in the traffic light, there are always just a few sites where the neighbor objects are closer to each other than two empty cells. In the traffic light, for example, each of the four blinkers is surrounded by twelve empty cells. Just two of these twelve empty cells are next nearest neighbors to two objects at the same time. The other ten are further away from the next nearest object.

These theoretical arguments and the observation of many simulations justify the assumption that most objects in the final steady state are separated by at least two empty cells. This assumption allows the theoretical prediction of a fractal box count dimensionality for small box sizes. The agreement between the results of the simulations with the results of the theoretical prediction shows that the assumption of a two cell excluded area around each object in the steady state is a good approximation (see Chapter 5.3.3).

The assumption of a two cell excluded area insures that no box with $\delta \leq 3$ will contain cells from two different objects at the same time. This fact makes it possible to

\footnotetext{
a It has been shown that there exist configurations in Life which have no predecessor. Such a configuration is called "Garden of Eden",
} 
predict $N(\delta)$ and the dimensionality $D_{s}$ for box sizes $1 \leq \delta \leq 3$. First, the average number of boxes needed to cover object $i, \bar{n}^{i}(\delta)$, is calculated:

$$
\bar{n}^{i}(\delta)=\frac{1}{n^{i}{ }_{t o t}(\delta)} \sum_{x} n^{i}{ }_{x}(\delta) x
$$

where $n^{i}{ }_{\text {tot }}(\delta)$ is the total number of possibilities to cover object $i$ and $n^{i}{ }_{x}(\delta)$ is the number of possibilities when the object covers exactly $x$ boxes. With the experimentally determined distribution $\alpha(i)$ (see Fig. 4) follows

$$
N(\delta)=\sum_{i=1}^{5} \alpha(i) \bar{n}^{i}(\delta),
$$

for $1 \leq \delta \leq 3$ (see Tab. 2 for the values of the calculations). The results of the calculations show a power-law relationship of $N(\delta)$ with $\delta$ (see Fig. 8). The slope in Fig. 8 represents the fractal box count dimensionality and was found to be $D_{s}=0.653$ (see Eqn. (5.2)).

Figure 8 also shows that the average result for the whole Life universe divides the individual objects into two classes. A line of alive cells, like the blinker, is a onedimensional object, a block of alive cells is a two-dimensional object. Therefore block and blinker show steeper slopes in Fig. 8 than the average for the final state of Life. The other objects, beehive, boat and burloaf, have characteristic holes of empty cells in them and demonstrate therefore a flatter slope in Fig. 8 than the average for the whole Life universe. 


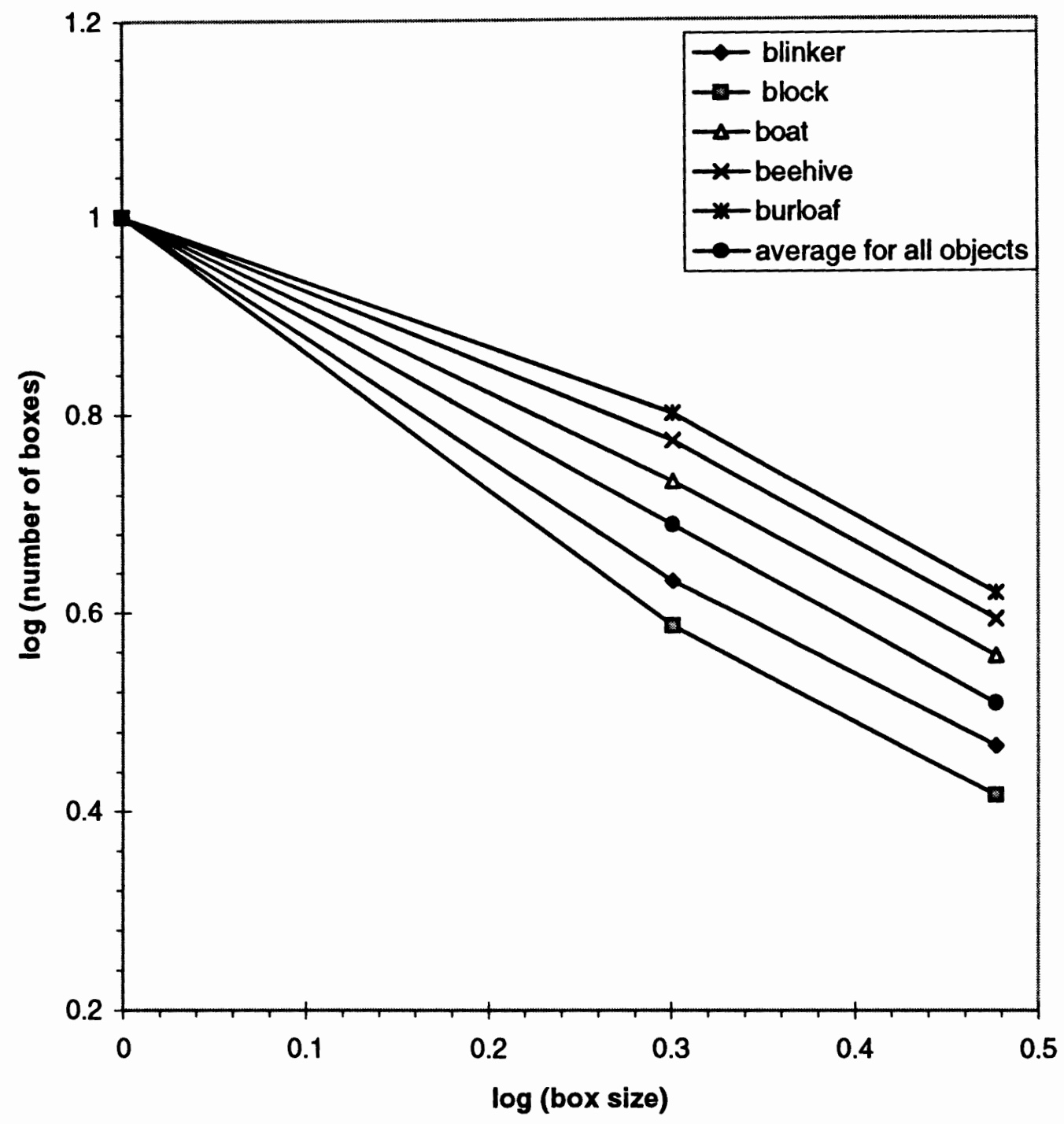

Fig. 8: Logarithm of the number of boxes needed to cover the individual objects and the "Life" average for all objects in the final state vs. the log of the box size. The number of covered boxes for box size one was normalized to ten for all objects to show the different relationships for each objects. 


\begin{tabular}{|c|c|c|c|c|c|}
\hline animal & $\delta$ & $n_{\text {tot }}^{i}(\boldsymbol{\delta})$ & $x$ & $n^{i}{ }_{x}(\delta)$ & $\bar{n}^{i}(\delta)$ \\
\hline \multirow{4}{*}{ blinker } & 1 & 1 & 3 & 1 & 3 \\
\hline & 2 & 4 & 2 & 4 & 2 \\
\hline & \multirow[t]{2}{*}{3} & \multirow[t]{2}{*}{9} & 1 & 3 & \multirow[t]{2}{*}{$15 / 9$} \\
\hline & & & 2 & 6 & \\
\hline \multirow{7}{*}{ block } & 1 & 1 & 4 & 1 & 4 \\
\hline & \multirow{3}{*}{2} & \multirow{3}{*}{4} & 1 & 1 & \multirow{3}{*}{$9 / 4$} \\
\hline & & & 2 & 2 & \\
\hline & & & 4 & 1 & \\
\hline & \multirow{3}{*}{3} & \multirow{3}{*}{9} & 1 & 4 & \multirow{3}{*}{$16 / 9$} \\
\hline & & & 2 & 4 & \\
\hline & & & 4 & 1 & \\
\hline \multirow{7}{*}{ boat } & 1 & 1 & 5 & 1 & 5 \\
\hline & \multirow[t]{2}{*}{2} & \multirow[t]{2}{*}{4} & 3 & 3 & \multirow[t]{2}{*}{$13 / 4$} \\
\hline & & & 4 & 1 & \\
\hline & \multirow{4}{*}{3} & \multirow{4}{*}{9} & 1 & 1 & \multirow{4}{*}{$22 / 9$} \\
\hline & & & 2 & 4 & \\
\hline & & & 3 & 3 & \\
\hline & & & 4 & 1 & \\
\hline \multirow{13}{*}{ beehive } & 1 & 1 & 6 & 1 & 6 \\
\hline & 2 & 4 & 4 & 4 & $16 / 4$ \\
\hline & \multirow{3}{*}{3} & \multirow{3}{*}{9} & 2 & 3 & \multirow{3}{*}{$26 / 9$} \\
\hline & & & 3 & 4 & \\
\hline & & & 4 & 2 & \\
\hline & \multirow{4}{*}{4} & \multirow{4}{*}{16} & 1 & 2 & \multirow{4}{*}{$38 / 25$} \\
\hline & & & 2 & 8 & \\
\hline & & & 3 & 4 & \\
\hline & & & 4 & 2 & \\
\hline & \multirow{4}{*}{5} & \multirow{4}{*}{25} & 1 & 6 & \multirow{4}{*}{$52 / 25$} \\
\hline & & & 2 & 13 & \\
\hline & & & 3 & 4 & \\
\hline & & & 4 & 2 & \\
\hline
\end{tabular}




\begin{tabular}{|c|c|c|c|c|c|}
\hline animal & $\delta$ & $n^{i}{ }_{t o t}(\boldsymbol{\delta})$ & $x$ & $n^{i}{ }_{x}(\boldsymbol{\delta})$ & $\bar{n}^{i}(\boldsymbol{\delta})$ \\
\hline \multirow{13}{*}{ burloaf } & $\overline{1}$ & 1 & 7 & 1 & 7 \\
\hline & \multirow[t]{2}{*}{2} & \multirow[t]{2}{*}{4} & 4 & 1 & \multirow[t]{2}{*}{$19 / 4$} \\
\hline & & & 5 & 3 & \\
\hline & \multirow[t]{2}{*}{3} & \multirow[t]{2}{*}{9} & 3 & 6 & \multirow[t]{2}{*}{$30 / 9$} \\
\hline & & & 4 & 3 & \\
\hline & \multirow{4}{*}{4} & \multirow{4}{*}{16} & 1 & 1 & \multirow{4}{*}{$43 / 16$} \\
\hline & & & 2 & 6 & \\
\hline & & & 3 & 6 & \\
\hline & & & 4 & 3 & \\
\hline & \multirow{4}{*}{5} & \multirow{4}{*}{25} & 1 & 4 & \multirow{4}{*}{$58 / 25$} \\
\hline & & & 2 & 12 & \\
\hline & & & 3 & 6 & \\
\hline & & & 4 & 3 & \\
\hline
\end{tabular}

Tab. 2 The number of possibilities to cover a object, $n_{\text {tot }}^{i}(s)$, the number of possibilities when a object covers exactly $x$ boxes, $n_{x}^{i}(s)$, and the average number of boxes covered for each object $i$ and box size $1 \leq s \leq 3$. 


\section{Dividing Scale for Small and Large Scale Regime}

The box size, at which the two-dimensional, random scale regime begins and the fractal small scale regime ends, is determined by the size and the average distance between two next nearest objects. We can determine the average distance between next nearest objects from the density and size of the objects. The average distance between objects should roughly be the dividing scale between the small and large scale regime. First, we determine the average size of the objects in the final steady state of Life:

$$
\bar{a}=\frac{\sum_{i=1}^{5} \alpha(i) a_{i}}{\sum_{i=1}^{5} a_{i}}
$$

where $a_{i}$ is the average number of alive cells for object $i$ and $\alpha(i)$ the distribution of the objects. Then, the average available "empty space" $\bar{A}$, the average number of alive cells plus the average number of empty cells around each object, is given by:

$$
\bar{A}=\frac{\bar{a}}{\rho_{\infty}}
$$

with $\rho_{\infty}=0.0285$ being the density of alive cell in the final steady state [4].

We assume, for reasons of simplicity, that this average space is a square. Then the average distance $I$ between objects is simply given by the square-root of the average empty space $\bar{A}$ around each object $i$ :

$$
l=\sqrt{\bar{A}} \cong 12.3
$$




\subsubsection{Experimental Box Count Results}

Computer simulations are carried out to determine the fractal box count dimensionality of the Game of Life in the final steady state. Automata with sizes $L$ of $78,99,120,160$, and 256 cells are observed. It usually took thousands of time steps to reach the final steady state starting from random initial conditions. Different automata sizes were used to examine if Life's dimensionality is a universal property and does not depend on the size of the automaton. Ten experiments were carried out for every automaton size. Initial percentages of alive cells were chosen between $20 \%$ and $40 \%$.

Figure 9 shows a $\log -\log$ plot of the density of occupied boxes $\rho(\delta)$ (see Eqn. (2.2)) vs. box size $\delta$ for five different lattice sizes and also the theoretical predicted curves for the two scale extremes. We find for $\log \rho(\delta)$ vs. $\log \delta$ a universal curve, all the values for the different lattice sizes fall on one curve. Because lattice size does not affect our results for the box count dimensionality, we claim that the correlations in the final state of Life are smaller than the lattice sizes examined in our simulations. Figure 9 shows further that the theoretical determined fractal dimensionality for $1 \leq \delta \leq 3$ is valid for the much larger scale regime $1 \leq \delta \leq 8$ and that $D_{l}=2$ is valid for $\delta \geq 16$. This result implies that the largest distances between next nearest objects are around 16 cells (see Chapter 5.3.2). $D_{s}=0.653$ is calculated for the slope of the predicted curve for $1 \leq \delta \leq 3$. We find experimentally $D_{s}^{\text {exp }}=0.658 \pm 0.005$ for box sizes $1 \leq \delta \leq 8$. 


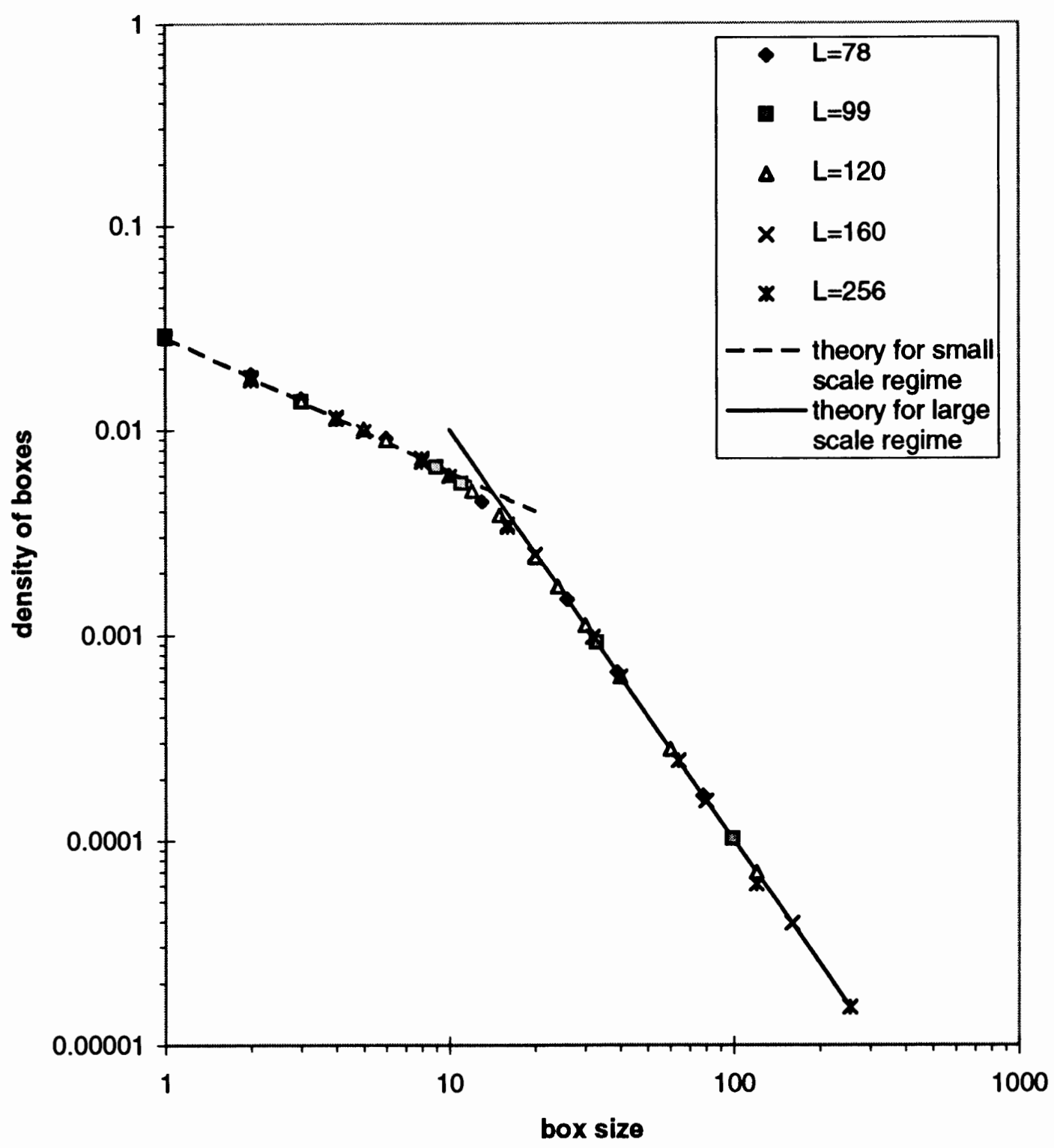

Fig 9: Log-log plot of the density of boxes $\rho(\delta) v s$. box size $\delta$. In addition, the theoretical predictions for the small scale regime and the large scale regime were plotted. 
The theoretical curves for the small and large scale regime were found to cross at box size $\delta_{c} \approx 13.2 \pm 0.8$. This is close to our prediction for the dividing scale between the two scale regimes $l_{c} \cong 12.3$ (see Chapter 5.3.2 C).

Figure 10 shows the time evolution of $\rho(\delta)$ vs. $\delta$ for a Life universe with $L=120$ and periodic boundary conditions. At time $\mathrm{T}=0$, the initial state, $30 \%$ alive cells are randomly distributed, therefore the curve follows Eqn. (5.6). Time $\mathrm{T}=25$ belongs to the initial period (see Chapter 4.2.1). This time period is characterized by a dynamics which seems chaotic. $\mathrm{T}=400$ belongs to the active period. In the active period parts of the Life universe have already settled down and they are populated by static and periodic objects. The regime with dimensionality two begins for box sizes $\delta \cong 16$, the same as for the final steady state of Life. This result implies that steady state areas, populated with still-lifes and oscillators, with sizes larger than 16 cells must exist already at $\mathrm{T}=400$.

Figure 11 shows $\rho(\delta)$ vs. $\delta$ for the final state of Life, and for $2.85 \%$ and $30 \%$ randomly distributed alive cells. Figure 11 shows the difference between randomly distributed alive cells and the final state of Life in the small scale regime. This difference is caused by the organization of alive cells into objects. 


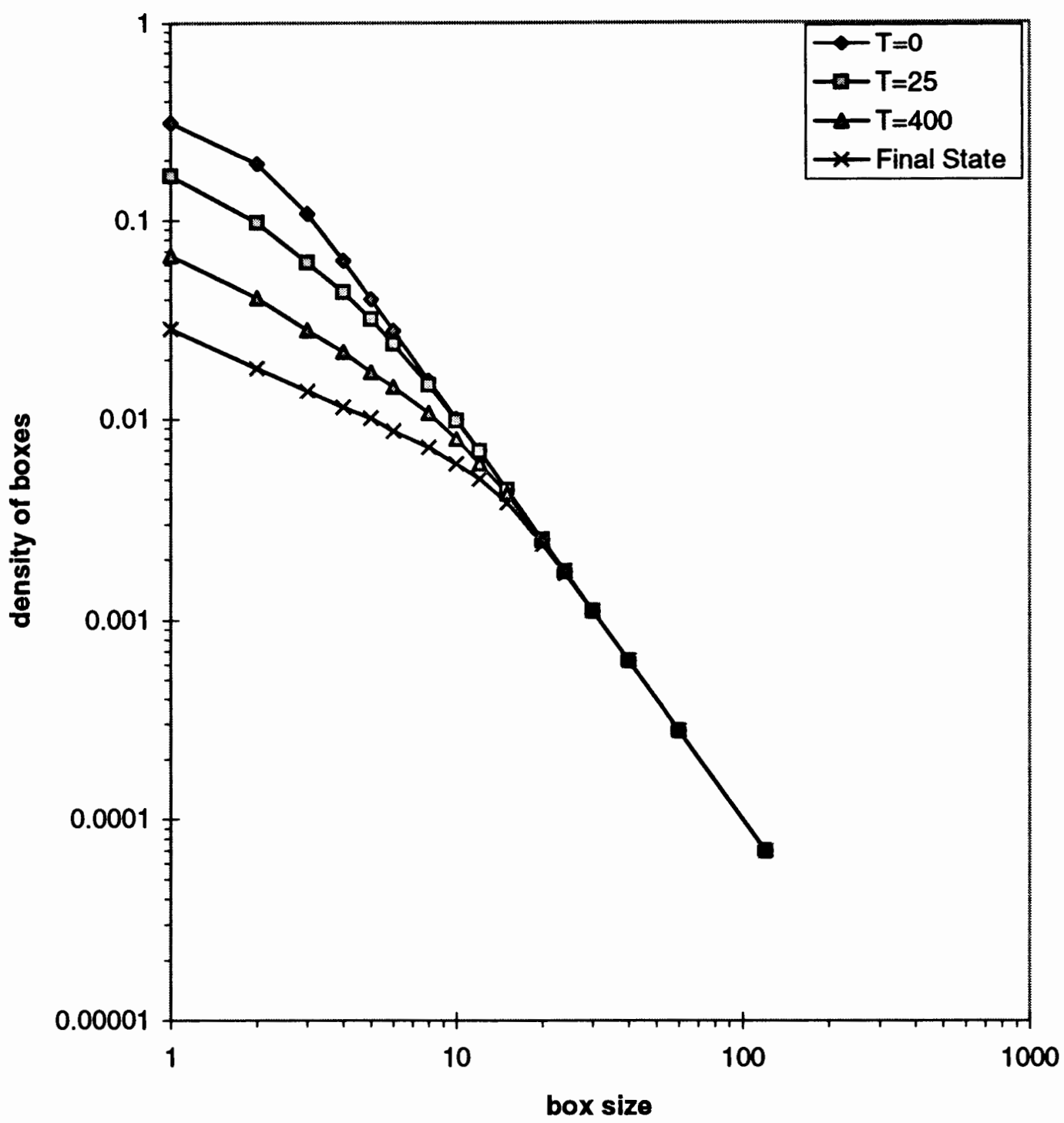

Fig 10: The density of boxes $\rho(\delta)$ vs. the box size $\delta$ at different time steps during the evolution of Life. $T=0$ is the initial state with $30 \%$ alive cells randomly distributed. $T=25$ belongs to the initial random regime. $T=400$ belongs to the active regime and the final state to the glider regime. These simulations were performed in a Life universe with $L=120$ and periodic boundary conditions. 


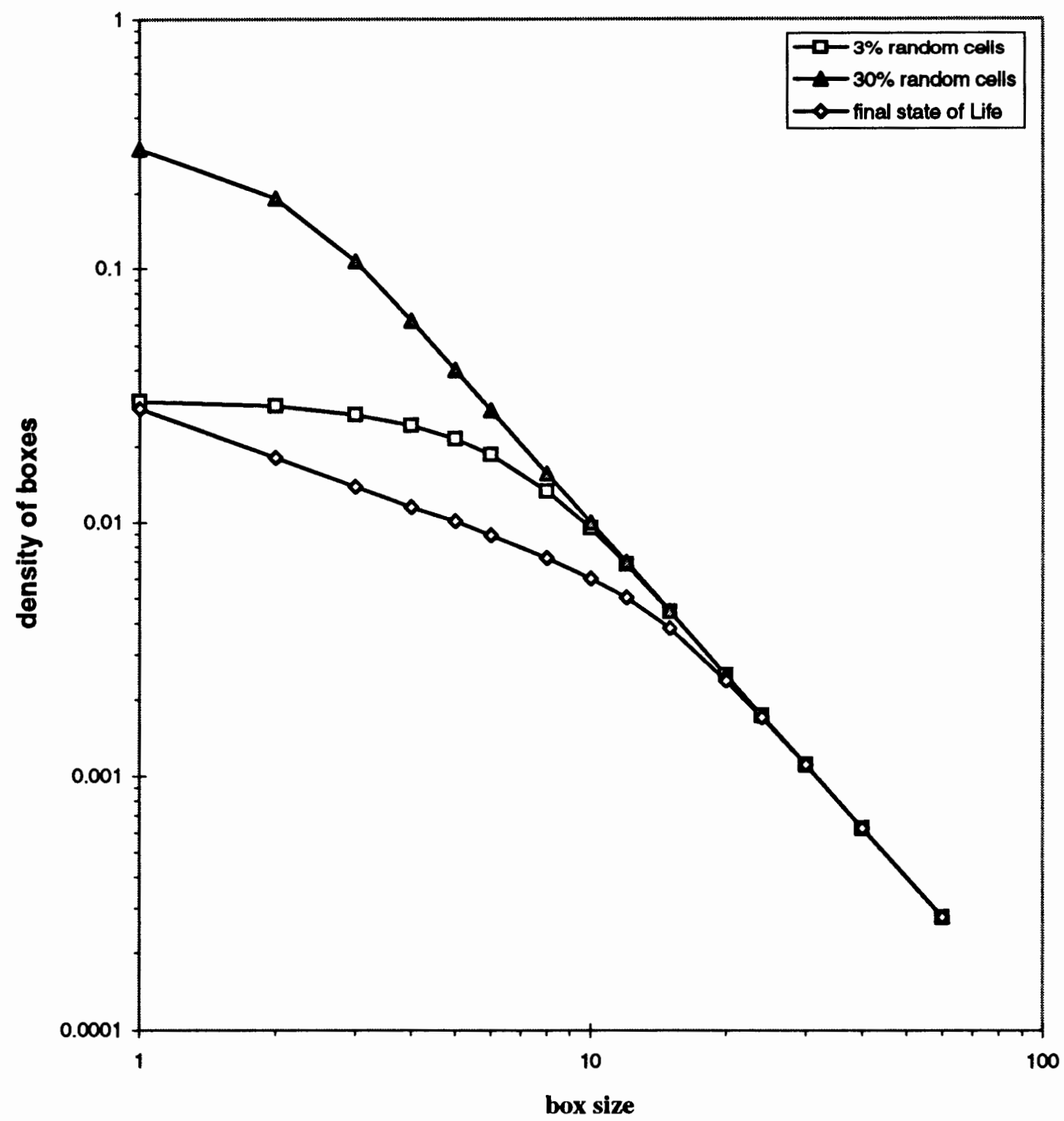

Fig. 11: The density of boxes $\rho(\delta)$ vs.box size $\delta$ for the final state of Life, the initial state with $30 \%$ randomly distributed alive cells, and for $3 \%$ randomly distributed alive cells. 


\subsection{Correlations between alive Cells}

We have pointed out before that one of the characteristics of critical phenomena is a long-range correlation between the elements of a system. We reported earlier that it was claimed that Life is a self-organized critical system, we therefore expect that Life exhibits long range correlations between alive cells. We use the pair correlation function from statistical mechanics to examine the correlations between alive cells. We deduce from Eqn. (3.5) for the pair-correlation function $\Gamma_{l}(r)$ between alive cells:

$$
\Gamma_{l}(r)=\Gamma_{l}\left(\left|\vec{r}_{i}-\vec{r}_{j}\right|\right)=\left\langle\left(s_{i}-\left\langle s_{i}\right\rangle\right)\left(s_{j}-\left\langle s_{j}\right\rangle\right)\right\rangle
$$

with $\langle\ldots\rangle$ being the average taken over the whole lattice. $r=|\vec{r}|=\left|\vec{r}_{i}-\vec{r}_{j}\right|$ is the distance between site $i$ and site $j$ with the position vectors $\vec{r}_{i}$ and $\vec{r}_{j}$. The "spin" values are $s_{i}=1$ if the cell at site $i$ is in the alive state, and $s_{i}=0$ if the cell is in the dead state. We find then:

$$
\Gamma_{l}(r)=\frac{1}{N} \sum_{i} s\left(\vec{r}_{i}+\vec{r}\right) s\left(\vec{r}_{i}\right)-\left\langle s\left(\vec{r}_{i}\right)\right\rangle^{2}
$$

where $N$ is the total number of lattice sites. Our goal is to determine the correlation of alive cells during the evolution of Life. $\Gamma_{l}(r)$ in Eqn. (5.14) is still dependent on the density of alive cells $\rho(t)$. Therefore the normalized correlation $\widetilde{\Gamma}_{l}(r)$ is introduced:

$$
\tilde{\Gamma}_{l}(r)=\frac{\Gamma_{l}(r)}{\Gamma_{l}(0)}=\frac{\Gamma_{l}(r)}{\rho(t)(1-\rho(t))} .
$$


Figure 12 shows the normalized correlation $\tilde{\Gamma}_{1}(r)$ in the different time periods of Life. The correlation is measured between cells lying on a vertical or horizontal line (see next section for discussion of dependence of $\tilde{\Gamma}_{1}(r)$ on direction of $\vec{r}$ ). We see in Figure 12 that already after 20 time steps, correlations between alive cells extend over a distance of five cells although the dynamics of Life looks very chaotic and the density of alive cells is $20 \%$. In the active period we find that the correlations extend on the average over distances up to $r \approx 15$. This is much larger than in the initial and the glider period (see Fig. 12). In the glider period, the inactive regions, populated by still-lifes, are dominant. The size of the five common objects in vertical and horizontal direction ranges from two to four cells (see Fig. 4). We can conclude from this and the fact that each object has a two cell excluded area around itself (see section 5.3.2 B), that $\widetilde{\Gamma}_{1}(r)$ is in the final steady state for $1 \leq r \leq 3$ only determined by the shapes of the different objects. $\widetilde{\Gamma}_{1}(r)$ decreases in the glider period for $r \leq 4$ and is even negative for $r=4$. This is caused by the two cell excluded area around each object. $\widetilde{\Gamma}_{1}(r)$ increases then again because of neighbor objects and reaches a local maximum for $r=6$. This local maximum is already visible in the active period because of the already extended steady state areas in this period.

We conclude from these results that the correlations between alive cells extend in Life never much farther than $r=15$ and that the largest correlations occur in the active period and not in the glider period. This is in agreement with our box count results. Therefore both, the correlation results and the box count results, do not 


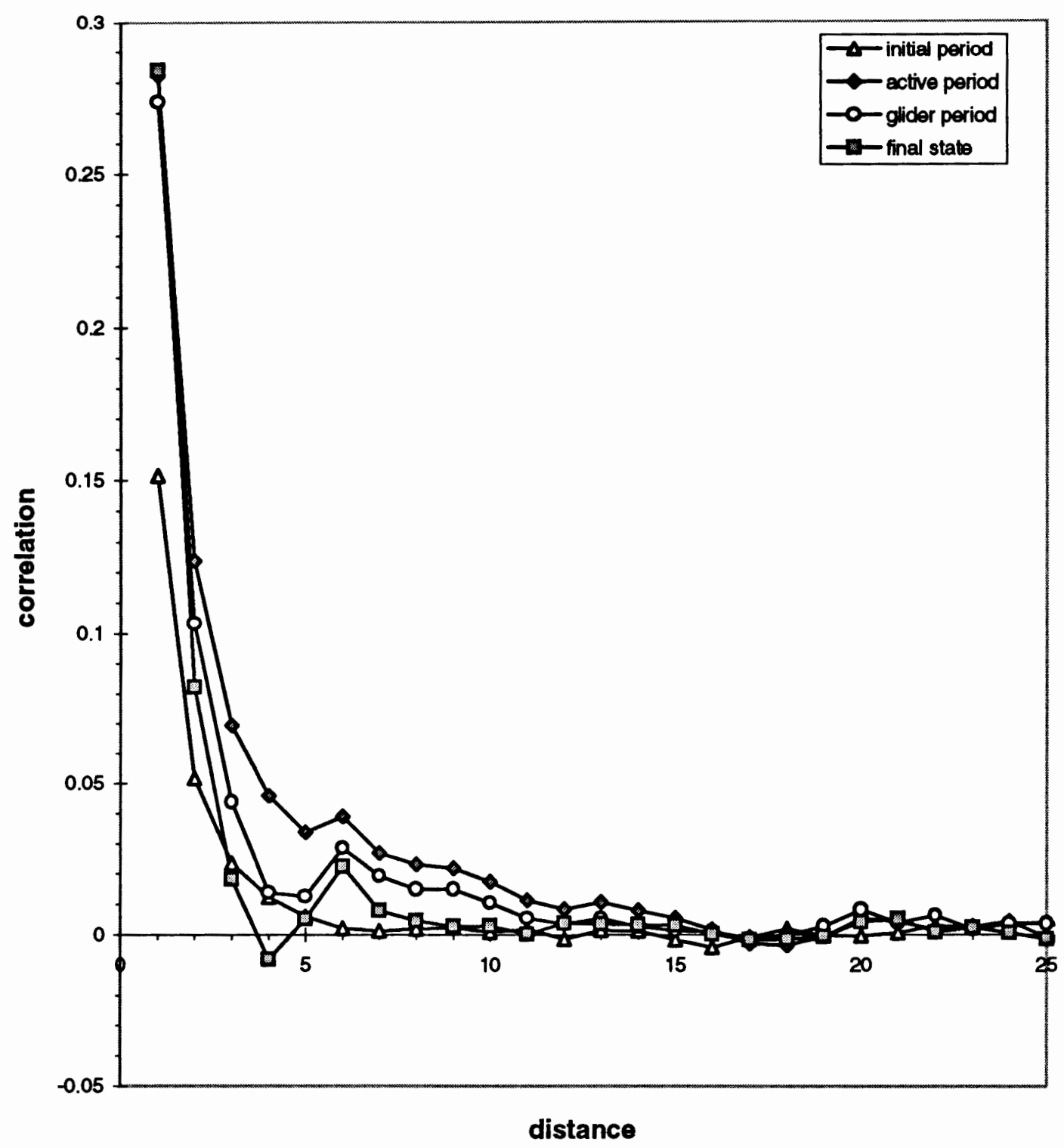

Fig. 12: The pair correlation function $\tilde{\Gamma}_{l}(r)$ for alive cells $v s$.distance $r$ in the different time periods of Life. The correlation was measured in vertical and horizontal direction. 
support the claim that Life is critical but rather that it is sub-critical. We will come back to this question in Chapter 5.6.

\subsection{Geometrical Properties of Life}

No thought has been given to the geometry of Life so far. One would probably suspect that Life has an eightfold rotational symmetry, because the transition rules of Life do not distinguish between any of the eight cells in the neighborhood template $N$. This would imply that the correlation $\widetilde{\Gamma}_{l}(r)$ is the same in these eight directions of $\vec{r}$.

So far all our correlation measurements were for cells lying on a horizontal or vertical line. Figure 13 shows the correlation $\tilde{\Gamma}_{l}(r)$ for cells in vertical/horizontal and in diagonal direction vs. distance $r$, measured in number of cells. The distance $r$ in Fig. 13 did not distinguish between vertical/horizontal and diagonal direction of $\bar{r}$. Figure 13 shows that $\tilde{\Gamma}_{l}(r)$ has the same qualitative features for both directions of $\vec{r}$, it decreases first and then increases again. But the minimum for $\tilde{\Gamma}_{l}(r)$ is reached in diagonal direction already for $r=3$ and the local maximum is reached in diagonal direction for $r=4$, whereas it is reached in the horizontal/vertical direction for $r=6$. A further difference is that $\tilde{\Gamma}_{l}(r)$ never becomes negative in the diagonal direction.

In Fig. 14 we plotted the pair correlation function for alive cells $\widetilde{\Gamma}_{l}\left(r_{e}\right)$ vs. the Euclidean distance $r_{e}$ for the vertical/horizontal and the diagonal direction. The Euclidean distance in horizontal/vertical direction is given by $r_{e}^{h / v}=r$, where $r$ is the 
number of cells separating the sites for which $\widetilde{\Gamma}_{l}\left(r_{e}\right)$ is measured. In diagonal direction the Euclidean distance $r_{e}^{d}$ is given by:

$$
r_{e}^{d}=r \sqrt{2}
$$

where $r$ is again the number of cells separating the sites for which $\tilde{\Gamma}_{l}\left(r_{e}\right)$ is measured. We find that $\widetilde{\Gamma}_{l}\left(r_{e}\right)$ coincides much better for the different directions than $\tilde{\Gamma}_{l}(r)$ does. We conclude from this result that although the transition rules do not distinguish between the different cells in the neighborhood template $N$, the pair correlation function $\tilde{\Gamma}_{l}(r)$ between cells does depend on the Euclidean distance between them.

We suggest that the reason for this lies in some of the special properties of a lattice with square cells. Each corner cell of the eight cell square neighborhood has a diagonal neighbor cell which is not neighbor to any of the other cells in the neighborhood. Further, a corner cell has five neighbor cells with a distance of two cells from the center cell. All the other neighborhood cells have just three neighbor cells at a distance of two from the center cell. Because the corner cells have more neighbors with a larger distance to the center cell, the correlations between the center cell and the corner cells are weaker than between the center cell and the rest of the neighborhood cells.

More simulations have to be performed to examine this claim. We think it would be interesting to investigate other lattices, like the hexagonal lattice, for similar properties. 


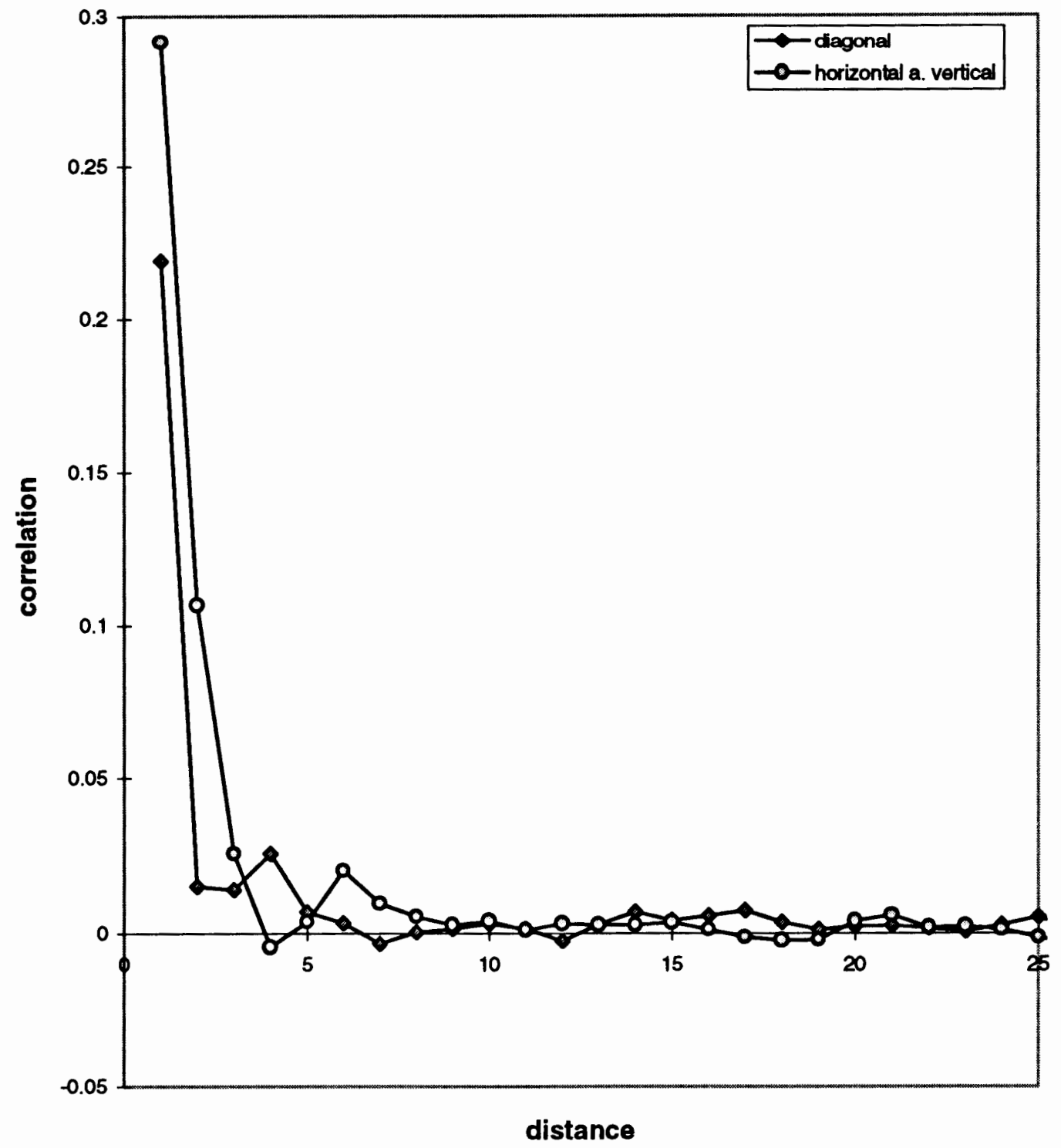

Fig. 13: Correlation $\tilde{\Gamma}_{l}(r)$ of alive cells in vertical/horizontal and diagonal direction $v$ s. distance $r$ in the final steady state of Life. 


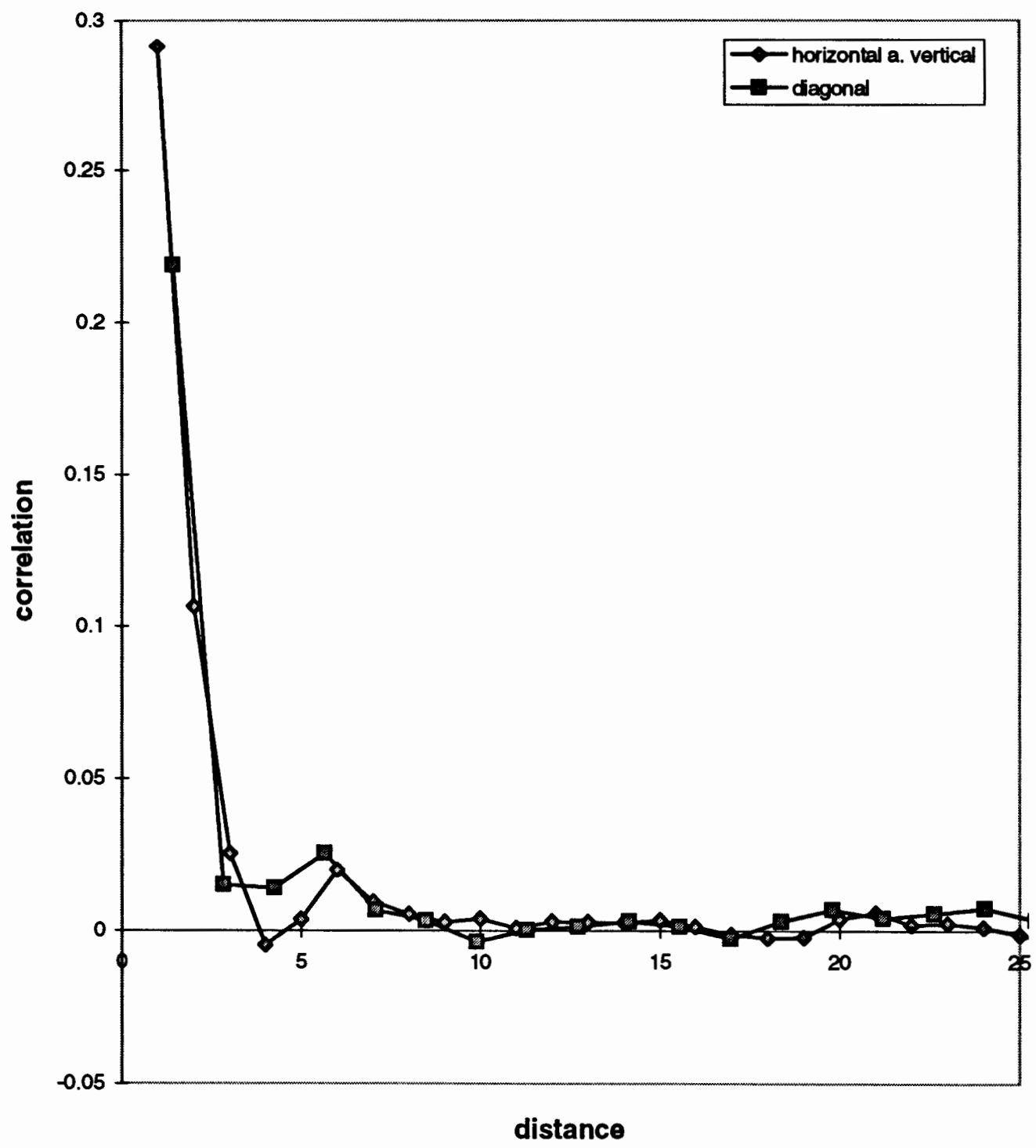

Fig. 14: $\tilde{\Gamma}_{l}\left(r_{e}\right)$ vs. the Euclidean distance $r_{e}$ for the horizontal/vertical and the diagonal direction. 


\subsection{Size-Distribution of Active Clusters}

In Chapter 3.5 we discussed self-organized criticality in general, and in Chapter 4.4 we presented the results which Bak used to support his claim that Life is a selforganized critical system. Bak disturbed the final steady state of Life at a random site and measured the response. He reported that the spatial and temporal activity, following a single perturbation, is distributed on a power-law. Bak also found that the activity is sustained on a fractal. Bak concluded from the fact that the activity does not decay or explode exponentially that life and death are highly correlated in time and space: the system has evolved into a critical state.

We want to point out once more that the following definitions and reported results which Bak used to support his claim seem somewhat arbitrary or unclear:

- Bak's definition of activity similar to the definition for the conservative sand-pile model is problematic because Life is a non-conservative model.

- Bak does not justify his definition of the total number of active sites as the sum over time and space of active sites.

- We could not determine if Bak had excluded periodic activity. Because he did not mention periodic activity explicitly, we assume that periodic activity was included. We argue that this would distort Bak's data because periodic activity is correlated over very short distances, namely the size of the periodic object. 
- Bak's description of self-organized criticality in Life is unclear. He claims that "the local structures of Life organize themselves into a critical state". Our results in Chapter 5.3 and 5.4 indicate that the local structures of Life are randomly distributed. The statistical distribution of the objects does not change after perturbations of the final steady state.

We presented in Chapter 5.4 our results for the correlation of alive cells. We reported that the correlation length is relatively short and decays in the glider period. We claimed that these results suggest that Life is sub-critical.

To investigate Bak's claim of self-organized criticality in Life further, we examined the size distribution of activity clusters in the active period and in the glider period. We want to mention again that it has been shown by Bagnoli et. al. (see Chapter 4.3) that $t_{c}$, the time where the active period ends and the glider period begins, is not lattice size dependent [24]. Therefore Bagnoli et. al concluded that if Life is indeed critical, the glider period should be the final state of Life for infinite lattice sizes and should also show characteristics of critical behavior.

We used in our examinations the following definitions for activity and active clusters::

- We define an active cell as a cell which has changed its state from the last time step excluding periodic state changes like the ones in the blinker. Periodic activity is excluded because a periodic object is in a final equilibrium state and the activity is only correlated to activity in the same object. 
- We define further an activity cluster as a configuration of active cells which are connected to each other as next nearest neighbors.

If activity in Life is sustained on a fractal, as Bak claims, we would expect that the activity clusters are distributed on a power-law, similar to the scale-free fluctuations occurring in a ferromagnet at the critical point. We examined size distribution of active clusters for different lattice sizes in the active and the glider period. Figure 15 shows a $\log -\log$ plot of the activity cluster size distribution $D_{a}(s)$ in the active (7.5\% alive cells) and in the glider period (3.8\% alive cells) for a lattice with $L=120$. The values plotted in Fig. 15 are normalized so that $D_{a}(s)=1$ for $s=1$. Figure 15 shows that $D_{a}(s)$ follows a power-law neither in the active nor in the glider period. It shows further that the behavior of $D_{a}(s)$ is very similar in the active and the glider period for cluster sizes up to $s=50$. For $s>50$ only a few activity clusters are found in the glider period whereas in the active period clusters with sizes up to $s=90$ are found. This indicates that over time the large activity clusters decay, but that the statistical distribution of activity clusters stays the same for smaller sizes.

We plotted in Fig. $16 D_{a}(s)$ for lattice sizes $L=80,120$, and 160 in the glider period. If the activity in Life were critical and sustained on a fractal, as claimed by Bak, we would expect that the upper cutoff for the cluster size is lattice size dependent. We find that $D_{a}(s)$ is not lattice size dependent. 


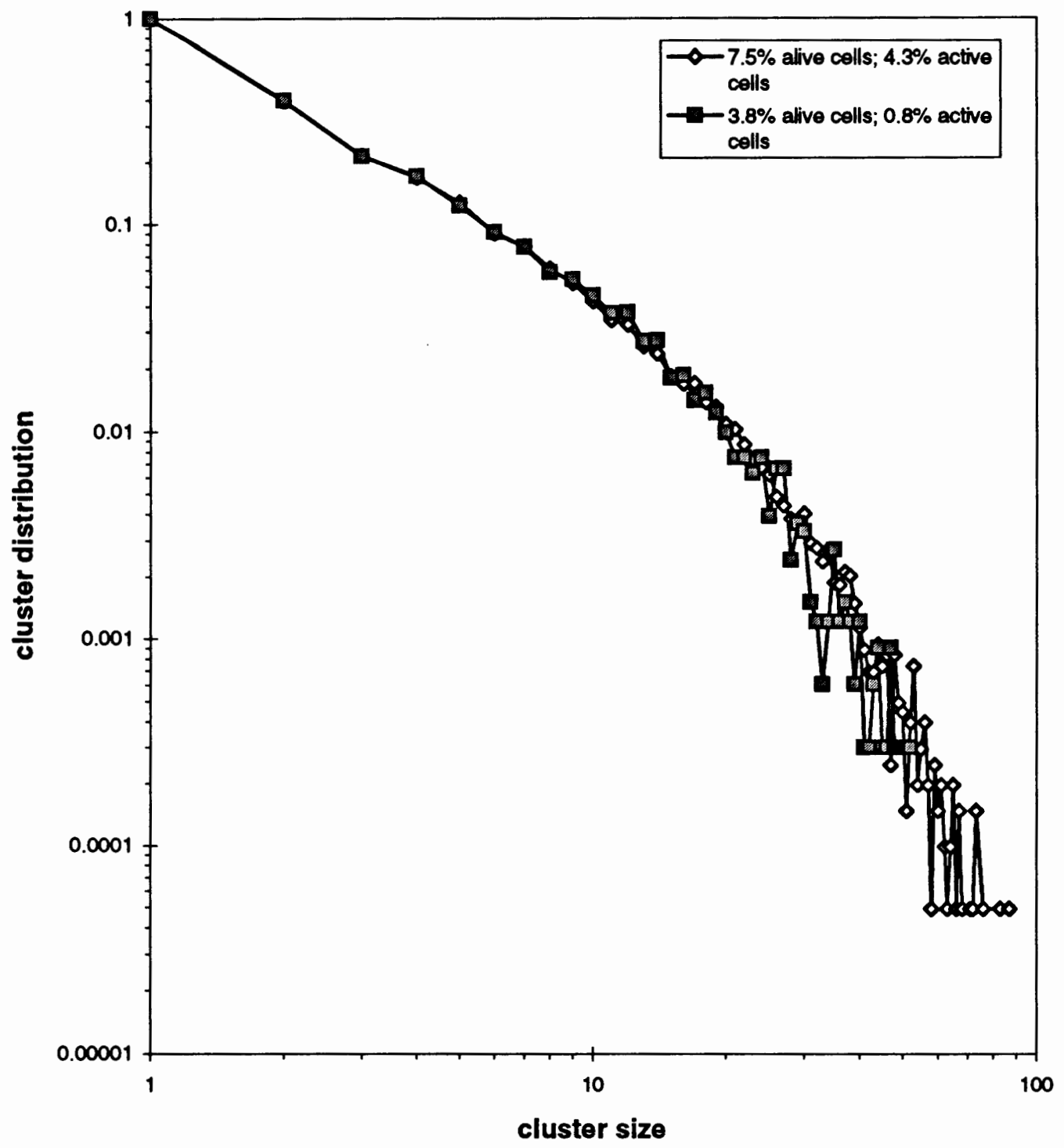

Fig. 15: This figure shows a $\log -\log$ plot of the activity cluster size distribution $D_{a}(s)$ in the active (7.5\% alive cells) and in the glider period (3.8\% alive cells) for a lattice with $L=120$. The plotted values are normalized so that $D_{a}(s)=1$ for $s=1$ 


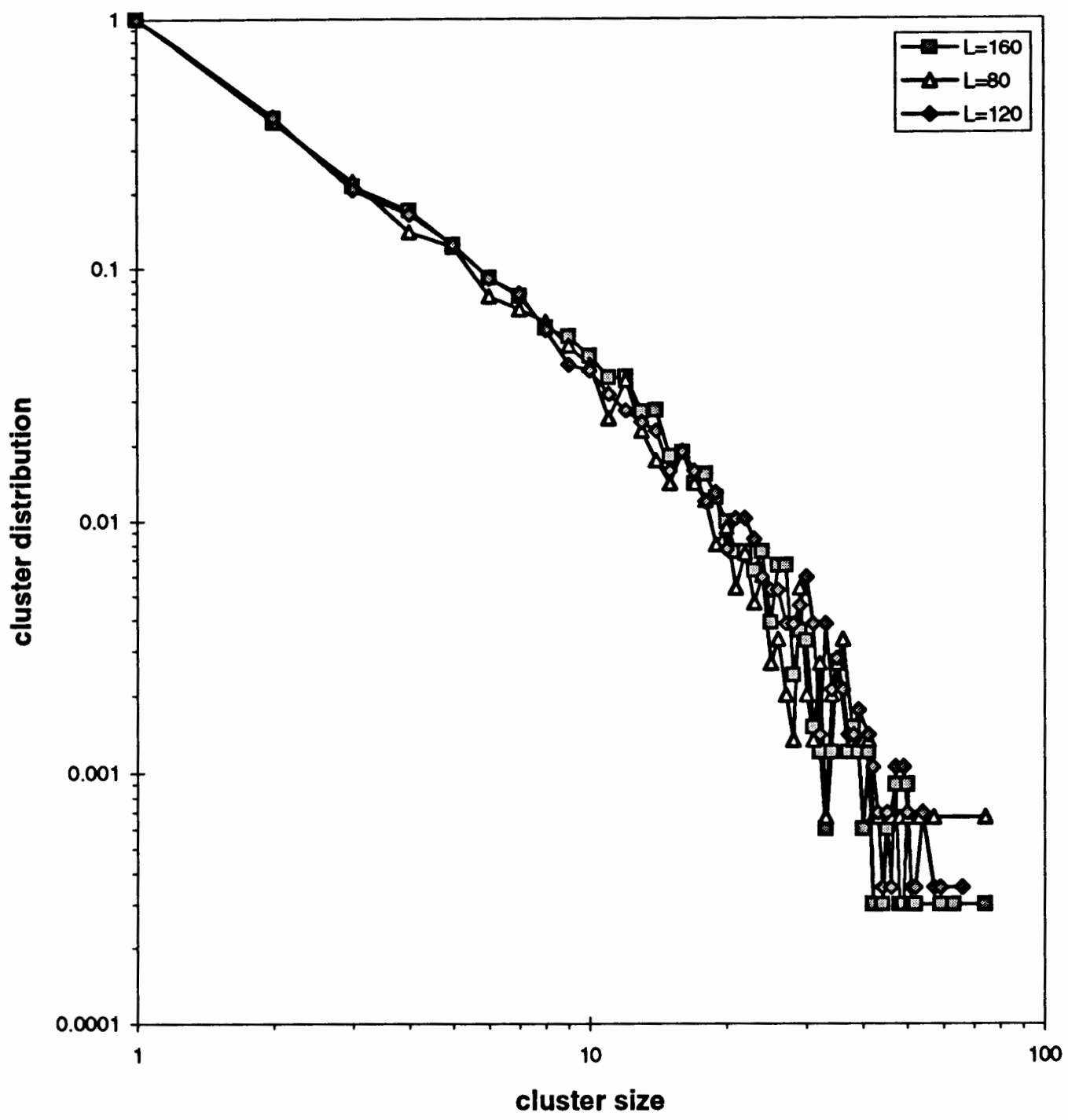

Fig. 16: This plot shows the activity cluster size distribution $D_{a}(s)$ for lattice sizes $L=80,120$, and 160 in the glider period. 
We conclude from the following results, (1) that the activity cluster size distribution does not follow a power-law, (2) that the size distribution does neither depend on the time period nor on the lattice size, and (3) that large activity clusters seem to vanish in the glider period, that activity in Life is not sustained on a fractal. We suggest that activity clusters in Life are randomly distributed with a certain size distribution, similar to the objects in Life. We claim that this would for most systems mean that they are sub-critical. But Life has propagating structures, like the glider, and we did not investigate their influence on the activity distribution. We also did not find any reported results on that matter. We suggest that more research needs to be done in this direction to clarify the meaning of gliders for criticality in Life.

\subsection{Summary of Results}

We have presented in this thesis the major findings and concepts in complexity research for investigating complex systems and in particular cellular automata. Further, we have summarized the most important findings for the complex cellular automaton Game of Life and have shown why it is considered to be the most fascinating cellular automaton. We also have reported the following new results about Life: First, we report the distribution of objects for the final steady state. We use then the box count method to investigate the final steady state of Life. We find two different regimes, a small scale regime for box sizes $1 \leq \delta \leq 8$ with a dimensionality $D_{s}^{\exp }=0.658 \pm 0.005$ 
and a large scale regime for $\delta \geq 16$ with a dimensionality $D_{l}=2$, which indicates that the final state of Life contains randomly distributed objects.

Further, we investigate the correlations between alive cells. We find the largest correlations in the active period with correlations extending up to a distance $r=15$. The correlations decrease in the glider period. The relatively short correlation length and the decreasing of the correlation in the glider period indicate that Life is probably sub-critical.

In addition, we report an interesting geometrical property of Life. We find that Life does not have an eightfold symmetry as expected from the transition rules. We find that the correlation of alive cells seems to relate closer to the Euclidean distance between cells than just to the distance in number of cells, as expected from the transition rules. We propose that this property is characteristic for the square lattice and suggest the investigation of other lattices, like the hexagonal lattice.

Last, we examine the size distribution of the activity clusters in Life. We find that the activity clusters have a fixed distribution law which is not influenced by the lattice size or the density of cells, except for big clusters. We investigate lattice sizes $\mathrm{L}=80,120$, and 160 . These results also suggest that Life is not critical. We want to point out again that the question if Life is critical or not is important for complexity research. It would support Langton's claim, which connects complexity, computation, and criticality, if Life were critical. On the other hand, one would have to rethink 
Langton's theory when such an almost "perfect candidate" for complex systems would not fit into it. 


\section{Bibliography}

[1] Philip W. Anderson, Science, 1972, 177 (393).

[2] Steven Weinberg, Dreams of a Final Theory, Vintage, 1992.

[3] M. Gardener, Sci.Am. 223 (4) (1997) 120; 223 (5) (1970) 118; 223 (6) (1970) $114 ; 224$ (2) (1971) 112.

[4] A.W. Burks, ed., Essays on Cellular Automata, (Univ. of Illinois Press, Urbana, IL, 1970).

[5] J. Von Neumann, A.W. Burks, ed., Theory of Self-reproducing Automata, (Univ. of Illinois Press, Urbana, IL, 1966).

[6] David G. Green, World Wide Web, http://www.csu.edu.au/complex_systems/tutoriall.html, 1993

[7] S. Wolfram, Universality and Complexity in Cellular Automata, Physica D 10 (1984) 1-35.

[8] C. G. Langton, Studying Artificial Life with Cellular Automata, Physica D 22 (1986) 120-149.

[9] C. G. Langton, Computation at the Edge of Chaos, Physica D 42 (1990) 12-37.

[10] P. Bak, C. Tang and K. Wiesenfeld, Phys. Rev. Lett. 59 (1987) 381.

[11] P. Bak, C. Tang and K. Wiesenfeld, Phys. Rev. A 38 (1988) 364.

[12] B. Mandelbrot, The Fractal Geometry of Nature (Freeman, San Francisco, 1982)

[13] P. Bak, K. Chen and C. Tang, Phys. Lett. A 147 (1990) 297

[14] P.Bak, Physica A 191 (1992) 41

[15] P. Bak, K. Chen, and M. Creutz, Nature 342 (1989) 780

[16] B. Drossel and F. Schwabl, Physica A 191 (1992) 47 
[17] M.D. Raup, Science 231 (1986) 528.

[18] S.A. Kauffmann and S. Johnson, J. Theoretical Biology 149 (1991) 467.

[19] E.R. Berlekamp, J.H. Conway and R.K. Guy, Winning Ways for your Mathematical Plays, Vol.2 (Academic Press, New York, 1982).

[20] P.W. Atkins, The Second Law (Freeman, New York, 1984).

[21] M. Gardner, Wheels, Life and Other Stories (Freeman, New York, 1983) chs. 2022

[22] C. Bennett and M.S. Bourzutschky, Nature 350 (1991) 468.

[23] Alstrom P., Physical Review E 49 (4) (1994) 2507

[24] F. Bagnoli, R. Rechtman and S. Ruffo, Physica A 171 (1991) 249

[25] W. Poundstone, The Recursive Universe, (William Morrow and Company, New York, 1985).

[26] T. Hwa and M.Kardar, Phys. Rev. Lett. 64 (1990) 1813.

[27] J.B.C. Garcia, M.A.F. Gomes, T.I. Jyh , T.I. Ren and T.R.M. Sales, Physica Review E 48 (1993) 3345

[28] J. Feder, Fractals (Plenum Press, New York, 1988) 